Research Article

\title{
Study on the Potential Mechanism of Fructus Tribuli in the Treatment of Hypertensive Vascular Remodeling Based on Network Pharmacology and Molecular Docking
}

\author{
Shuyue Wang, ${ }^{1}$ Fei Guo, ${ }^{2}$ Xiaochen Sun, ${ }^{1}$ Xiao Song, ${ }^{1}$ Yaohui Yuan, ${ }^{1}$ Chao Zhang $\mathbb{D}^{1}$, \\ Guitao Lin, ${ }^{1}$ and Huagang Sheng $\mathbb{1}^{1}$ \\ ${ }^{1}$ School of Pharmaceutical Sciences, Shandong University of Traditional Chinese Medicine, Jinan 250355, China \\ ${ }^{2}$ The Affiliated Hospital of Shandong University of Traditional Chinese Medicine, Jinan 250011, China \\ Correspondence should be addressed to Chao Zhang; tougaotcm@163.com and Huagang Sheng; shenghuagang@sdutcm.edu.cn
}

Received 13 August 2020; Revised 9 December 2020; Accepted 23 December 2020; Published 8 January 2021

Academic Editor: Daniela Rigano

Copyright (C) 2021 Shuyue Wang et al. This is an open access article distributed under the Creative Commons Attribution License, which permits unrestricted use, distribution, and reproduction in any medium, provided the original work is properly cited.

\begin{abstract}
Background. Hypertensive vascular remodeling (HVR) is the pathophysiological basis of hypertension, which is also an important cause of vascular disease and target organ damage. Treatment with Fructus Tribuli (FT), a traditional Chinese medicine, has a positive effect on HVR. However, the pharmacological mechanisms of FT are still unclear. Therefore, this study aimed to reveal the potential mechanisms involved in the effects of FT on HVR based on network pharmacology and molecular docking. Materials and Methods. We selected the active compounds and targets of FT according to the Traditional Chinese Medicine Systems Pharmacology Database and Analysis Platform (TCMSP) and the Swiss Target Prediction database, and the targets of HVR were collected from the Online Mendelian Inheritance in Man (OMIM), GeneCards, and DrugBank databases. The protein-protein interaction network (PPI) was established using the STRING database. Moreover, Gene Ontology (GO) and Kyoto Encyclopedia of Genes and Genomes (KEGG) pathway analyses and network analysis were performed to further explore the potential mechanisms. Finally, molecular docking methods were used to evaluate the affinity between the active compounds and the main target. Results. Seventeen active compounds of FT and 164 potential targets for the treatment of HVR were identified. Component-target and PPI networks were constructed, and 12 main active components and 33 main targets were identified by analyzing the topological parameters. Additionally, GO analysis indicated that the potential targets were enriched in 483 biological processes, 52 cellular components, and 110 molecular functions. KEGG analysis revealed that the potential targets were correlated with 122 pathways, such as the HIF-1 signaling pathway, ErbB signaling pathway, and VEGF signaling pathway. Finally, molecular docking showed that the 12 main active components had a good affinity for the top five main targets. Conclusion. This study demonstrated the multiple compounds, targets, and pathway characteristics of FT in the treatment of HVR. The network pharmacology method provided a novel research approach to analyze potential mechanisms.
\end{abstract}

\section{Introduction}

Hypertension has become one of the most threatening public health problems worldwide [1]. It is the leading cause of death from cardiovascular and cerebrovascular diseases. Hypertensive vascular remodeling (HVR) is an adaptive change in the arterial structure and function. It is a selfprotective response to trauma and internal and external pressure changes to meet the physiological needs under different pathological conditions [2]. It mainly includes thickening of the vessel wall, a change in the wall-to-cavity ratio, and looseness of the small artery structures, resulting in abnormal vascular function. Basic and clinical studies on hypertension showed that vascular remodeling was accompanied by hypertension, was the pathophysiological basis [3], and formed a vicious circular causal mechanism with hypertension, which was also an important cause of vascular disease and target organ damage [4]. Reducing or reversing vascular remodeling has important theoretical significance and potential clinical application value for the 
early prevention and treatment of hypertension and its cardiac, brain, and renal complications $[5,6]$. Therefore, it is particularly important and urgent to search for targets that affect the vascular structure and research and discover therapeutic drugs that are safe and effective in controlling HVR.

Traditional Chinese medicine (TCM) has high activity and low toxicity in preventing and treating HVR, playing an irreplaceable role. Fructus Tribuli (FT), which is the dried fruit of Tribulus terrestris L. [7], is renowned in TCM as Ji- Li and has been used for thousands of years in Asian countries. Several previous investigations focused on its pharmacological activities demonstrated that FT improved sexual function [8], could be used to prevent and treat cardiovascular diseases [9], had neuroprotective [10] and memory improvement activity [11], and provided antidiabetic [12], antidepression [13], anti-inflammatory [14], and antioxidant effects [15].

In recent years, the efficacy of FT in HVR has attracted more and more attention. Zhang et al. found that the furostanol saponins from FT could inhibit the expression of myocardial transforming growth factor and connective tissue growth factor in ventricular muscle, reduce systolic blood pressure, and inhibit myocardial fibrosis in spontaneously hypertensive rats (SHRs). Thus, vascular remodeling induced by hypertension was significantly ameliorated [16]. Guo et al. showed that FT could reduce blood pressure and inhibit aortic vascular remodeling in elderly SHRs. The primary mechanisms involved are thought to be reduced collagen content in the thoracic aorta and regulation of the $\mathrm{NF}-\kappa \mathrm{B}$ signaling pathway [17]. Jiang et al. found that FT demonstrated antihypertensive and endothelial protective effects by regulating Erk2, FAK, and NF- $\kappa$ B p65 [18]. In addition, several clinical studies demonstrated that the Chinese patent medicine Xin-Nao-Shu-Tong, with extracts of FT as the active compounds, had a reliable antihypertensive effect [19]. However, its mechanism has not been fully elucidated. Thus, further comprehensive and systematic investigations are necessary.

TCM has the characteristics of integrity and diversity and can play a comprehensive role in multilevel, multilink, multitarget, and bidirectional regulation. Network pharmacology is a new field that integrates systems biology, omics, and computational biology to reveal the mechanism of drug action from an overall perspective and possesses integrity, synergistic effects, and dynamic characteristics [20]. This research thinking is consistent with the characteristics of the simultaneous effects of the multiple components of TCM [21]. Therefore, this study aimed to reveal the possible molecular mechanisms involved in the effects of FT on HVR based on a network pharmacology approach.

\section{Materials and Methods}

2.1. Collection of Active Compounds of FT. The chemical information about FT was obtained from the Traditional Chinese Medicine Systems Pharmacology Database and Analysis Platform (TCMSP, https://tcmspw.com/tcmsp. php), which is considered a special platform for Chinese herbal medicine based on systems pharmacology that provides the relationships between drugs, targets, and diseases, as well as comprehensive data on absorption, distribution, metabolism, and excretion (ADME) properties for each compound [22]. To screen for active compounds, oral bioavailability $(\mathrm{OB}) \geq 30 \%$ and drug-likeness $(\mathrm{DL}) \geq 0.18$ included in the ADME system were selected as the screening criteria by referring to the most common criteria in the TCMSP database $[23,24]$. In addition, by consulting the literature, we added several active compounds, which were removed by ADME screening or not recruited by the TCMSP database but have some potential value in the treatment of HVR. Then, the primary molecular formula of all active compounds was double-checked in the PubChem database (https://pubchem.ncbi.nlm.nih.gov/).

2.2. Screening of Targets Related to Active Compounds. The TCMSP and Swiss Target Prediction (http://www. swisstargetprediction.ch/) database were applied to identify the potential targets related to the active compounds of FT. Swiss Target Prediction is a web server for potential drug target prediction. Then, each of the predicted targets was inputted into the UniProt database (https://www.uniprot. org/) with "Homo sapiens" selected as the organism. After deleting repeated and nonhuman targets, we obtained the targets for each active compound.

2.3. Screening of Targets for HVR. Targets associated with HVR were retrieved from the Online Mendelian Inheritance in Man (OMIM, http://www.omim.org/) database, the GeneCards database (https://www.genecards.org/), and the DrugBank database (https://www.drugbank.ca/). OMIM is a comprehensive research resource of human genes and genetic diseases [25]. GeneCards is an integrative database, which provides information on all annotated and predicted human genes [26]. The DrugBank is a powerful bioinformatics database containing information on drugs, diseases, and their mechanisms of action and targets [27]. All of these databases are comprehensive, freely available online tools and can provide a relatively comprehensive overview of research results.

\subsection{Construction of the Target Protein-Protein Interaction} Network. To identify the potential targets of FT in the treatment of HVR, we intersected the active compound targets and disease targets. The common targets were considered to be potential targets. The obtained common targets were imported into STRING (https://string-db.org, Version 11.0) to perform protein-protein interaction (PPI) analysis. STRING is a database that predicts direct and indirect interaction and builds networks between proteins and proteins [28]. The data were saved in a tab-separated value (TSV) format and imported into Cytoscape 3.6.1 software where the PPI was drawn and analyzed [29]. We calculated the topological parameters of the network using the plug-in of "Network Analyzer." We then used degree, betweenness, and closeness as the three main parameters for 
critical target screening [30]. The Molecular Complex Detection (MCODE) plug-in was used to screen the densely connected regions and for cluster analysis of the PPI network [31]. We selected significant cluster modules from the constructed PPI network using MCODE. The degree of protein association in the module was scored using the following criteria: degree cutoff $=2$, node score cutoff $=0.2$, $\mathrm{K}$-score $=2$, and max depth $=100$.

2.5. Gene Ontology and Pathway Analysis. All potential targets were utilized for Gene Ontology (GO) enrichment analysis and Kyoto Encyclopedia of Genes and Genomes (KEGG) pathway enrichment analysis using the DAVID database (https://david.ncifcrf.gov/, Version 6.8). DAVID can provide systematic, comprehensive biological functional annotations for large-scale gene or protein lists, including biological processes (BP), cellular components (CC) molecular functions (MF), and pathways. In the GO and KEGG analyses, the lower the $P$ value, the stronger the correlation between the pathway and target; the higher the count, the more targets in the pathway. A threshold $P$ value of $<0.05$ was considered statistically significant.

\subsection{Construction of the Compound-Target-Pathway Network.} To further explore the pharmacological mechanisms of FT in the signaling pathway for treating HVR, the predicted target genes of each compound, the disease-related genes, and the KEGG pathway involved were all imported into Cytoscape software to establish a combinatorial network. In the network, the nodes represent compounds, targets, and pathways, while the edges represent the interaction between the nodes.

2.7. Molecular Docking. The molecular structures of the major active compounds in the Mol2 file format obtained from the TCMSP in the simulation description format (SDF) downloaded from the PubChem database or in the canonical simplified molecular-input line-entry specifications (SMILES) were imported into the Discovery Studio Client software for modification. The RCSB PDB database (http:// www.rcsb.org/) was searched to download the major target protein structure and import it into Discovery Studio Client for preprocessing such as deleting the water molecules and preparing the protein. The original ligand position was used as the active center to expand a certain range, and the amino acid residues in this range were defined as the active site. The default settings were used for the other settings. The receptor-ligand interaction module was used for molecular docking. The software finds the optimal binding conformation by locating the active sites of small molecule compounds and proteins and calculates and scores the affinity between the receptors and the ligands. LibDock scores between the compound and protein higher than the docking score between the ligand and the protein indicate that the component has a good affinity for the target. Finally, PyMOL software was used to visualize the docking results of the major active compound with their corresponding higherscoring protein.

\section{Results}

3.1. Screening of Active Compounds and Related Targets. Fifty-one compounds were screened by searching the TCMSP. Twelve compounds were collected using the screening conditions of $\mathrm{OB} \geq 30 \%$ and $\mathrm{DL} \geq 0.18$. Furthermore, previous studies reported that steroidal saponins are one of the main compounds of FT, which can be used to treat cardiovascular diseases $[32,33]$, so we added several steroidal saponins that were deleted because of the screening conditions or not recruited by the TCMSP. Finally, 17 active FT compounds were selected, which are shown in Table 1. The targets related to active compounds were predicted by the TCMSP and Swiss Target Prediction database. After combining the retrieval results and removing the duplicated targets, a total of 164 potential targets were obtained.

3.2. Screening of Targets of FT in the Treatment of HVR. In this study, we used three internationally recognized databases of disease genes. To improve the accuracy of the targets, the three database results were intersected using the Venny platform [34]. A total of 1152 targets were retrieved, as shown in Figure 1(a). By intersecting the target of HVR with that of FT, a total of 164 overlapping target genes were identified, which we considered potential targets of FT for treating HVR, as shown in Figure 1(b).

3.3. Construction of the Compound-Target Interaction Network. The interactions of the abovementioned 17 active compounds and 164 potential targets were defined as data and type files in Excel file format, and the relationship was visualized by Cytoscape software. Then, we constructed the compound-target interaction network, as shown in Figure 2. The diamonds represent the active compounds, and the circles represent their targets. A total of 181 nodes and 425 edges were obtained. The network topological parameters were analyzed using the Network Analyzer plug-in. The larger the node, the greater the degree value. There were 12 compounds with degrees $\geq 10$. MOL008559 and MOL008569 were exceptions with no connected targets. The topological parameters of the major active compounds are shown in Table 2, suggesting that these 12 compounds may be the major active compounds of FT in treating HVR, including kaempferol, isorhamnetin, (2aR,2'S,4R,4'R,5'S,6aS,6bS,8aS,8bR,9S,11aR,12aR,12bR)-4,4'dihydroxy-5',6a,8a,9-tetramethylicosahydro- $1 \mathrm{H}$-spiro [pentale no[2,1-a]phenanthrene-10,2'-pyran]-8(2H)-one, (Z)-3-(4-hydroxy-3-methoxy-phenyl)-N- [2-(4-hydroxyphenyl)ethyl]acrylamide, terrestriamide, and tigogenin.

3.4. Construction and Analysis of the PPI Network. The 164 common targets were uploaded to the STRING database to obtain the PPI network, and the results were saved as a TSV file and imported into Cytoscape software for processing to make the information more intuitive, as shown in Figure 3. 
TABLE 1: Detailed information on 17 active compounds from FT.

\begin{tabular}{|c|c|c|c|c|c|}
\hline Number & Molecule ID & Molecule name & $\begin{array}{c}\text { Molecule } \\
\text { weight }\end{array}$ & $\begin{array}{l}\text { OB } \\
(\%)\end{array}$ & $\mathrm{DL}$ \\
\hline FT1 & MOL000354 & Isorhamnetin & 316.28 & 49.60 & 0.31 \\
\hline FT2 & MOL000359 & Sitosterol & 414.79 & 36.91 & 0.75 \\
\hline FT3 & MOL000422 & Kaempferol & 286.25 & 41.88 & 0.24 \\
\hline FT4 & MOL000483 & $\begin{array}{l}\text { (Z)-3-(4-hydroxy-3-methoxy-phenyl)-N-[2-(4-hydroxyphenyl)ethyl]acrylamide } \\
\text { (2aR,2'R,4R,6aR,6bS,8aS,8bR,11aS,12aR,12bR)-4-((S)-2-(2,6-dimethylphenyl) }\end{array}$ & 313.38 & 118.35 & 0.26 \\
\hline FT5 & MOL008559 & $\begin{array}{c}\text { propoxy)-5',5',6a,8a-tetramethyl-8-methylenedocosahydro-1H-spiro[pentaleno[2,1- } \\
\text { a]phenanthrene-10,2'-pyran] }\end{array}$ & 573.00 & 59.49 & 0.28 \\
\hline FT6 & MOL008563 & $\begin{array}{c}\text { (3R,8S,9S,10R,13R,14R,17S)-17-((2S,5R)-5-ethyl-6-methylheptan-2-yl)-3-hydr } \\
\text { oxy-10,13-dimethyl-3,4,8,9,10,11,12,13,14,15,16,17-dodecahydro-1H-cyclopenta[a] } \\
\text { phenanthren-7(2H)-one }\end{array}$ & 428.77 & 40.93 & 0.79 \\
\hline FT7 & MOL008567 & $\begin{array}{c}\text { (3R,7R,8S,9S,10S,13R,14S,17R)-17-((2R,5S)-5-ethyl-6-methylheptan-2-yl)-3,10- } \\
\text { dimethyl-2,3,4,7,8,9,10,11,12,13,14,15,16,17-tetradecahydro-1H-cyclopenta[a] } \\
\text { phenanthren-7-ol }\end{array}$ & 414.79 & 34.21 & 0.76 \\
\hline FT8 & MOL008568 & (Z)-3-(3,4-dihydroxyphenyl)-N-[2-(4-hydroxyphenyl)ethyl]acrylamide & 299.35 & 113.25 & 0.24 \\
\hline FT9 & MOL008569 & $\beta$-sitosterol- $\beta$-D-glucopyranoside & 398.79 & 32.41 & 0.71 \\
\hline FT10 & MOL008588 & Terrestriamide & 327.36 & 114.09 & 0.29 \\
\hline FT11 & MOL008590 & $\begin{array}{c}\text { (2aR,2'S,4R,4'R,5'S,6aS,6bS,8aS,8bR,9S, } 11 \mathrm{aR}, 12 \mathrm{aR}, 12 \mathrm{bR})-4,4 \text { '-dihydroxy-5',6a,8a,9- } \\
\text { tetramethylicosahydro-1H-spiro[pentaleno[2,1-a]phenanthrene-10,2'-pyran]- } \\
8(2 \mathrm{H}) \text {-one }\end{array}$ & 444.72 & 58.74 & 0.76 \\
\hline FT12 & MOL008593 & 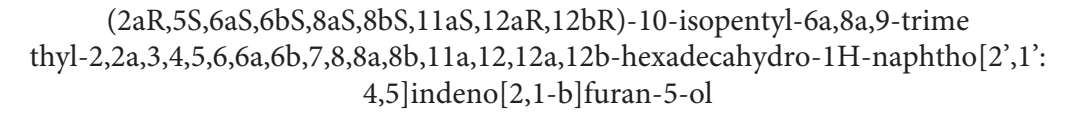 & 400.71 & 39.21 & 0.84 \\
\hline FT13 & MOL007291 & Hecogenin & 430.69 & 15.86 & 0.79 \\
\hline FT14 & MOL008581 & Tigogenin & 416.71 & 13.83 & 0.81 \\
\hline FT15 & 78177919 & Terrestrosin D & 1049.16 & - & - \\
\hline FT16 & 122169314 & Terrestrosin $\mathrm{K}$ & 1079.18 & - & - \\
\hline FT17 & - & Terrestroside B & 1312.63 & - & - \\
\hline
\end{tabular}

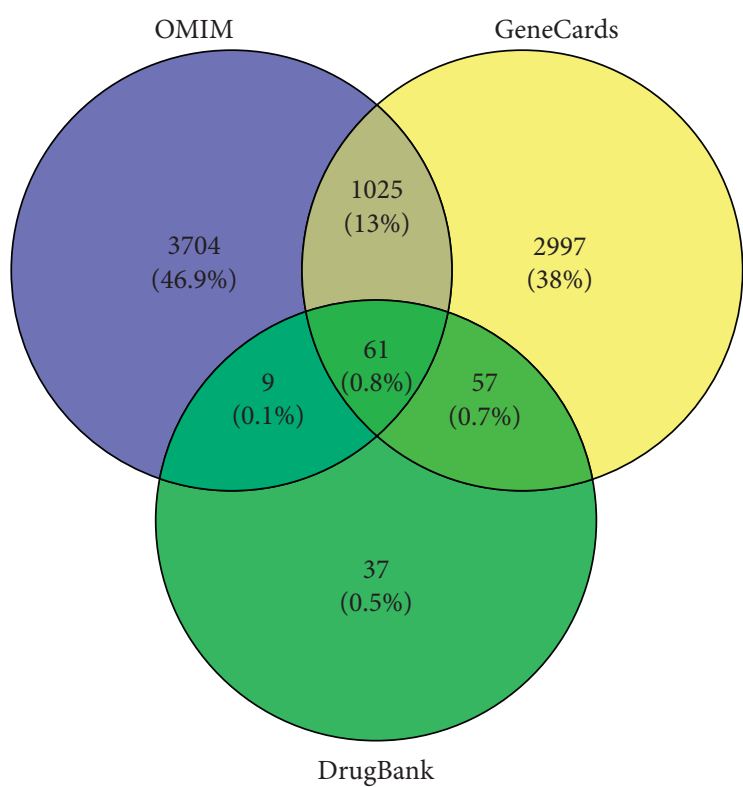

(a)

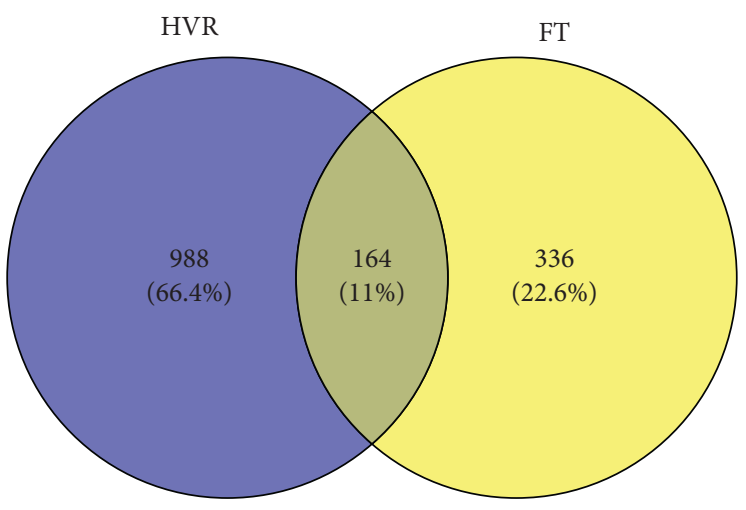

(b)

FIgURE 1: Venn diagram of related targets of FT in treating HVR. (a) The purple circle represents the targets of HVR from OMIM. The yellow circle represents the targets of HVR from GeneCards. The green circle represents the targets of HVR from DrugBank. (b) The purple circle represents the targets of HVR. The yellow circle represents the targets of the active compounds from FT.

The network diagram contained a total of 163 nodes and 2599 edges in which MERTK was not correlated with the other proteins and was, therefore, not included in the network. The proteins with greater degrees are shown as larger nodes and brighter color, and the edges with greater combined scores are shown by thicker and brighter lines. 


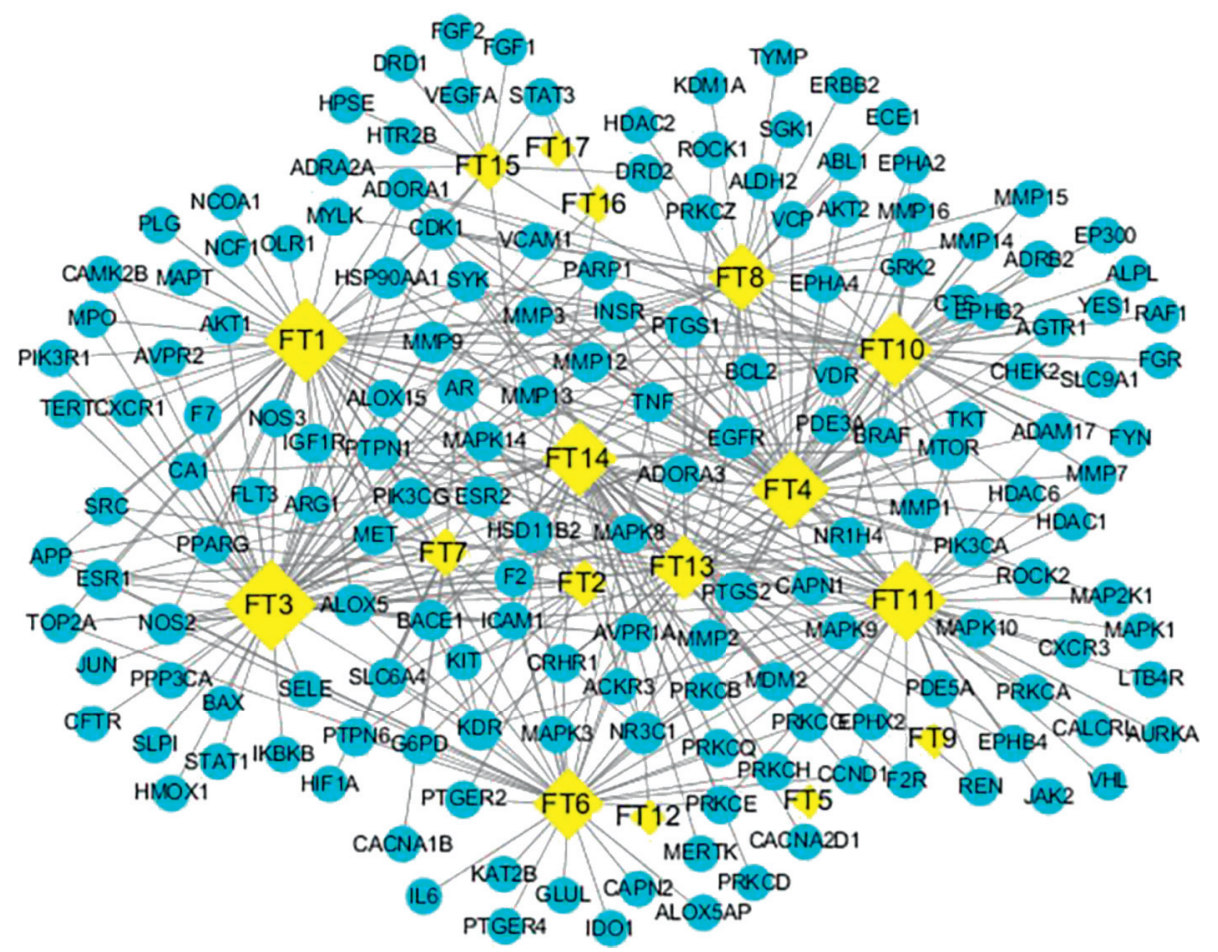

Figure 2: Compound-target interaction network. The yellow nodes represent the compounds, the blue nodes represent the targets, and the edges represent the interaction between them.

TABLE 2: Topological parameters of the major active compounds in the compound-target network.

\begin{tabular}{|c|c|c|c|c|}
\hline Number & Molecule ID & Betweenness centrality & Closeness centrality & Degree \\
\hline FT3 & MOL000422 & 0.23 & 0.43 & 57 \\
\hline FT1 & MOL000354 & 0.17 & 0.42 & 50 \\
\hline FT11 & MOL008590 & 0.19 & 0.42 & 47 \\
\hline FT4 & MOL000483 & 0.14 & 0.41 & 44 \\
\hline FT10 & MOL008588 & 0.14 & 0.40 & 41 \\
\hline FT14 & MOL008581 & 0.14 & 0.40 & 41 \\
\hline FT6 & MOL008563 & 0.14 & 0.40 & 37 \\
\hline FT8 & MOL008568 & 0.12 & 0.39 & 33 \\
\hline FT13 & MOL007291 & 0.07 & 0.38 & 30 \\
\hline FT7 & MOL008567 & 0.03 & 0.35 & 14 \\
\hline FT2 & MOL000359 & 0.02 & 0.36 & 13 \\
\hline FT15 & 78177919 & 0.09 & 0.35 & 13 \\
\hline
\end{tabular}

Targets whose betweenness centrality, closeness centrality, and degree all exceed the average values were selected as the major targets. The average degree in the network was 31.8896 , the average closeness centrality was 0.5334 , and the average betweenness centrality was 0.0056 . Thirty-three main targets met the screening conditions. The specific information is shown in Table 3. Among them, AKT1 had the highest degree value, which can interact with 111 proteins, followed by MAPK3, VEGFA, SRC, and IL6, which can interact with 108, 106, 102, and 99 proteins, respectively.

At the same time, the MCODE plug-in in Cytoscape software was used to analyze the PPI network. Nine significant clusters were obtained, as shown in Figure 4. Among them, the most significant, cluster 1 , contained 45 major target nodes including the top targets in the PPI network, which further demonstrated the importance of AKT1, MAPK3, VEGFA, SRC, and IL6. The clustering details are shown in Table 4.

3.5. GO and KEGG Pathway Enrichment Analysis. The 164 common targets were inputted into the DAVID database for GO and KEGG analysis. Of them, 645 GO terms were ascertained, including $483 \mathrm{BP}$ terms, $52 \mathrm{CC}$ terms, and 110 MF terms. The top 20 enriched GO terms were partially displayed by a bar chart according to the $-\log 10$ ( $P$ value), and the count of each term is also displayed in Figure 5. The results showed that the BPs were correlated with platelet activation, protein phosphorylation, peptidyl-serine phosphorylation, etc. The major CCs included the plasma membrane, cytosol, membrane raft, etc. The targets of MF 


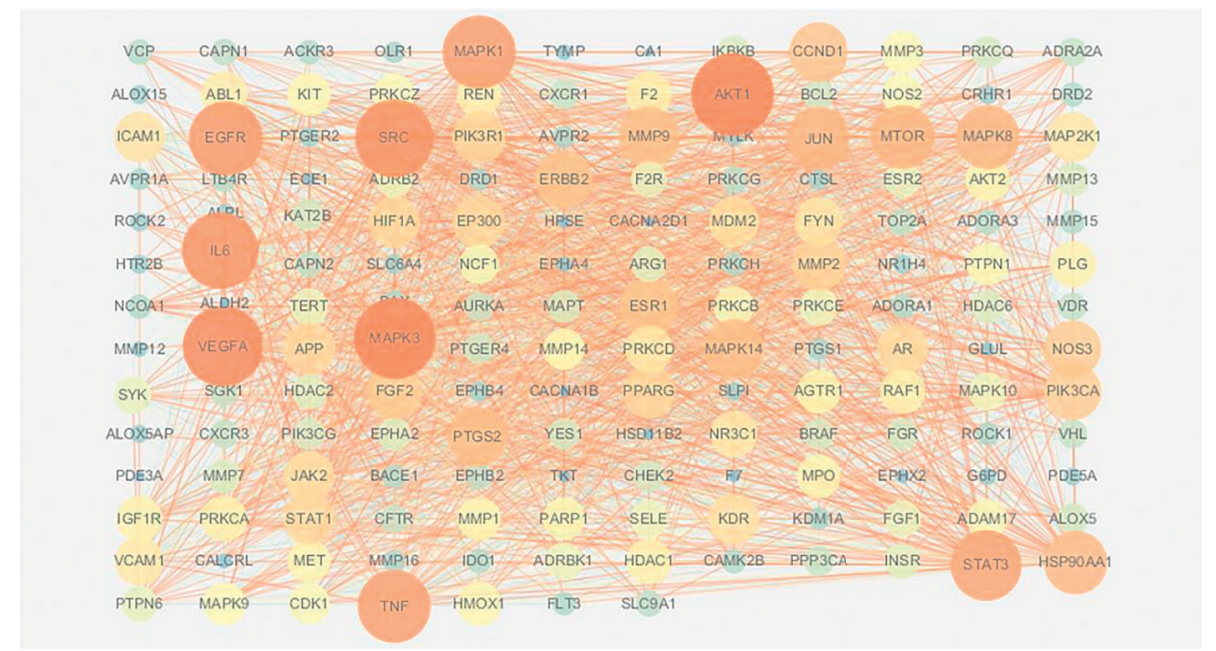

FIGURE 3: PPI network of FT in the treatment of HVR. The proteins with higher degrees are shown by larger nodes and brighter colors, and the edges with a greater combined score are shown by thicker and brighter lines.

TABLE 3: Topological parameters of the main targets of FT in the treatment of HVR.

\begin{tabular}{|c|c|c|c|c|}
\hline Gene names & Protein names & $\begin{array}{l}\text { Betweenness } \\
\text { centrality }\end{array}$ & $\begin{array}{l}\text { Closeness } \\
\text { centrality }\end{array}$ & Degree \\
\hline AKT1 & RAC-alpha serine/threonine-protein kinase & 0.07 & 0.75 & 111 \\
\hline MAPK3 & Mitogen-activated protein kinase 3 & 0.06 & 0.75 & 108 \\
\hline VEGFA & Vascular endothelial growth factor A & 0.06 & 0.73 & 106 \\
\hline SRC & Proto-oncogene tyrosine-protein kinase Src & 0.04 & 0.73 & 102 \\
\hline IL6 & Interleukin-6 & 0.05 & 0.71 & 99 \\
\hline EGFR & Epidermal growth factor receptor & 0.04 & 0.70 & 94 \\
\hline MAPK1 & Mitogen-activated protein kinase 1 & 0.03 & 0.70 & 93 \\
\hline TNF & Tumor necrosis factor & 0.03 & 0.68 & 92 \\
\hline STAT3 & Signal transducer and activator of transcription 3 & 0.02 & 0.68 & 88 \\
\hline MAPK8 & Mitogen-activated protein kinase 8 & 0.01 & 0.64 & 75 \\
\hline MTOR & Serine/threonine-protein kinase mTOR & 0.02 & 0.64 & 75 \\
\hline JUN & Transcription factor AP-1 & 0.01 & 0.64 & 74 \\
\hline HSP90AA1 & Heat shock protein HSP 90 -alpha & 0.02 & 0.64 & 74 \\
\hline MMP9 & Matrix metalloproteinase- 9 & 0.01 & 0.62 & 69 \\
\hline MAPK14 & Mitogen-activated protein kinase 14 & 0.01 & 0.62 & 68 \\
\hline PTGS2 & Prostaglandin $\mathrm{G} / \mathrm{H}$ synthase 2 & 0.01 & 0.62 & 68 \\
\hline CCND1 & G1/S-specific cyclin-D1 & 0.01 & 0.62 & 67 \\
\hline ESR1 & Estrogen receptor & 0.01 & 0.61 & 64 \\
\hline ERBB2 & Receptor tyrosine-protein kinase erbB-2 & 0.01 & 0.61 & 64 \\
\hline PIK3CA & $\begin{array}{c}\text { Phosphatidylinositol 4,5-bisphosphate 3-kinase catalytic subunit alpha } \\
\text { isoform }\end{array}$ & 0.01 & 0.61 & 62 \\
\hline NOS3 & Nitric oxide synthase, endothelial & 0.02 & 0.62 & 62 \\
\hline FGF2 & Fibroblast growth factor 2 & 0.01 & 0.60 & 59 \\
\hline PIK3R1 & Phosphatidylinositol 3-kinase regulatory subunit alpha & 0.01 & 0.59 & 55 \\
\hline PPARG & Peroxisome proliferator-activated receptor gamma & 0.01 & 0.59 & 54 \\
\hline EP300 & Histone acetyltransferase p300 & 0.01 & 0.59 & 54 \\
\hline STAT1 & Signal transducer and activator of transcription 1-alpha/beta & 0.01 & 0.59 & 53 \\
\hline APP & Amyloid-beta precursor protein & 0.02 & 0.59 & 53 \\
\hline PRKCD & Protein kinase $\mathrm{C}$ delta type & 0.01 & 0.56 & 44 \\
\hline NR3C1 & Glucocorticoid receptor & 0.02 & 0.57 & 43 \\
\hline $\mathrm{F} 2$ & Prothrombin & 0.01 & 0.56 & 41 \\
\hline PRKCA & Protein kinase $\mathrm{C}$ alpha type & 0.01 & 0.56 & 41 \\
\hline HDAC1 & Histone deacetylase 1 & 0.01 & 0.56 & 40 \\
\hline REN & Renin & 0.01 & 0.56 & 38 \\
\hline
\end{tabular}




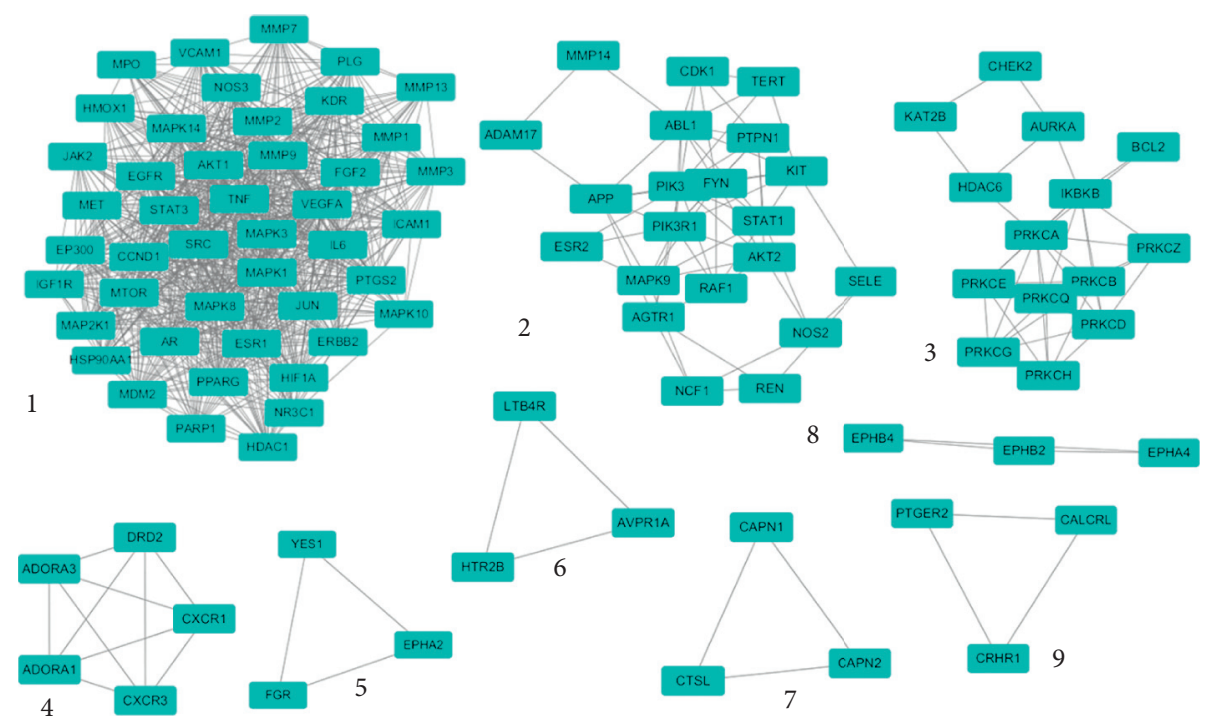

FIgURE 4: Clustering analysis results of the PPI network node. The blue nodes represent the targets, and the edges represent the interaction between them.

TABLE 4: Clustering analysis results of the PPI network node.

\begin{tabular}{|c|c|c|c|c|}
\hline Cluster & Genes & Score & Nodes & Edges \\
\hline 1 & $\begin{array}{c}\text { MDM2, EGFR, EP300, HDAC1, MPO, MAPK10, PLG, MMP9, SRC, MMP2, ERBB2, PPARG, MMP3, } \\
\text { STAT3, CCND1, JAK2, PARP1, KDR, PTGS2, VEGFA, TNF, VCAM1, MTOR, MAP2K1, MMP1, NOS3, } \\
\text { AKT1, AR, ICAM1, MAPK3, MAPK14, MMP7, FGF2, NR3C1, IGF1R, MAPK1, MAPK8, HMOX1, } \\
\text { ESR1, HIF1A, JUN, IL6, MMP13, MET, HSP90AA1 }\end{array}$ & 34.182 & 45 & 752 \\
\hline 2 & $\begin{array}{c}\text { NCF1, REN, NOS2, FYN, ADAM17, STAT1, PIK3CA, PIK3R1, KIT, CDK1, PTPN1, MMP14, SELE, } \\
\text { TERT, RAF1, MAPK9, ABL1, AKT2, AGTR1, APP, ESR2 }\end{array}$ & 6.500 & 21 & 65 \\
\hline 3 & $\begin{array}{c}\text { PRKCE, PRKCB, PRKCD, PRKCA, BCL2, PRKCQ, AURKA, PRKCG, CHEK2, HDAC6, KAT2B, } \\
\text { PRKCH, PRKCZ, IKBKB }\end{array}$ & 6.000 & 14 & 39 \\
\hline 4 & CXCR1, CXCR3, ADORA3, ADORA1, DRD2 & 5.000 & 5 & 10 \\
\hline 5 & CALCRL, PTGER2, CRHR1 & 3.000 & 3 & 3 \\
\hline 6 & LTB4R, AVPR1A, HTR2B & 3.000 & 3 & 3 \\
\hline 7 & CTSL, CAPN2, CAPN1 & 3.000 & 3 & 3 \\
\hline 8 & ЕРНВ4, ЕРНВ2, ЕРНА4 & 3.000 & 3 & 3 \\
\hline 9 & CALCRL, PTGER2, CRHR1 & 3.000 & 3 & 3 \\
\hline
\end{tabular}

mainly involved enzyme-binding, protein kinase activity, and ATP binding.

KEGG pathway annotation showed that the 164 common targets were involved in 122 pathways with $P$ values of less than 0.05 . The top 20 signaling pathways were listed in a bubble chart and are shown in Figure 6. The size of the dot reflects the number of targets in the analysis, and the different dot colors indicate the different $P$ values. The enrichment pathways of FT in treating HVR were mainly concentrated in the HIF-1 signaling pathway, ErbB signaling pathway, VEGF signaling pathway, TNF signaling pathway, FoxO signaling pathway, Fc epsilon RI signaling pathway, Rap1 signaling pathway, and the cAMP signaling pathway.

3.6. Compound-Target-Pathway Network Analysis. The active compounds, potential targets, and signal pathways were imported into Cytoscape software for visual processing to construct a compound-target-pathway network. Figure 7 shows that one active compound could correspond to multiple targets, and one target could correspond to multiple active compounds and multiple pathways. The network diagram fully reflected the characteristics of the synergistic relationships between the multiple components, targets, and pathways of FT.

3.7. Major Active Compound-Main Target Molecular Docking. To further analyze and verify the target-compound interactions, the top five main targets of AKT1, MAPK3, VEGFA, SRC, and IL6, which had higher degrees, were selected for molecular docking with the 12 major active compounds of FT. The LibDock score of the 12 small molecule compounds was obtained, as shown in Table 5. The component had a good affinity for the target when the LibDock score between the compound and protein was higher than the score between the ligand and protein. This table indicated that each of the 12 active compounds had one or more docking proteins with a high score, and VEGFA could bind to most compounds. The details of the docking simulation of the 


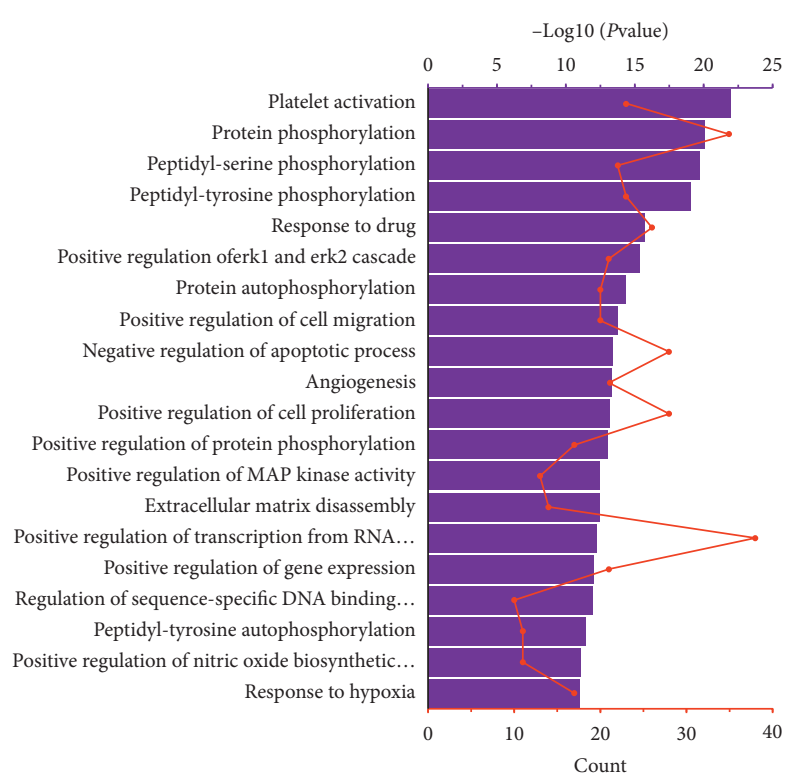

(a)

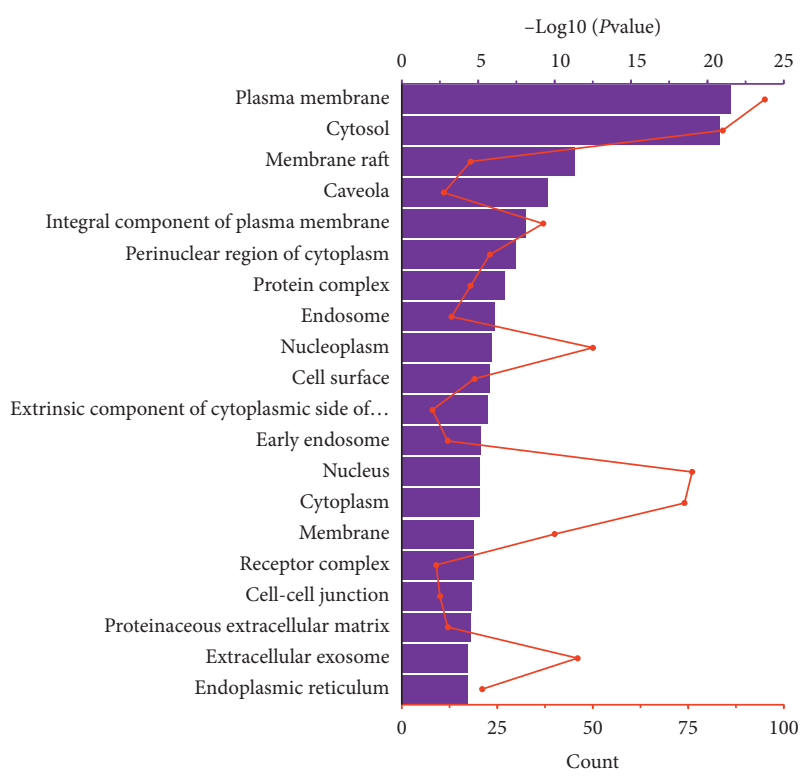

(b)

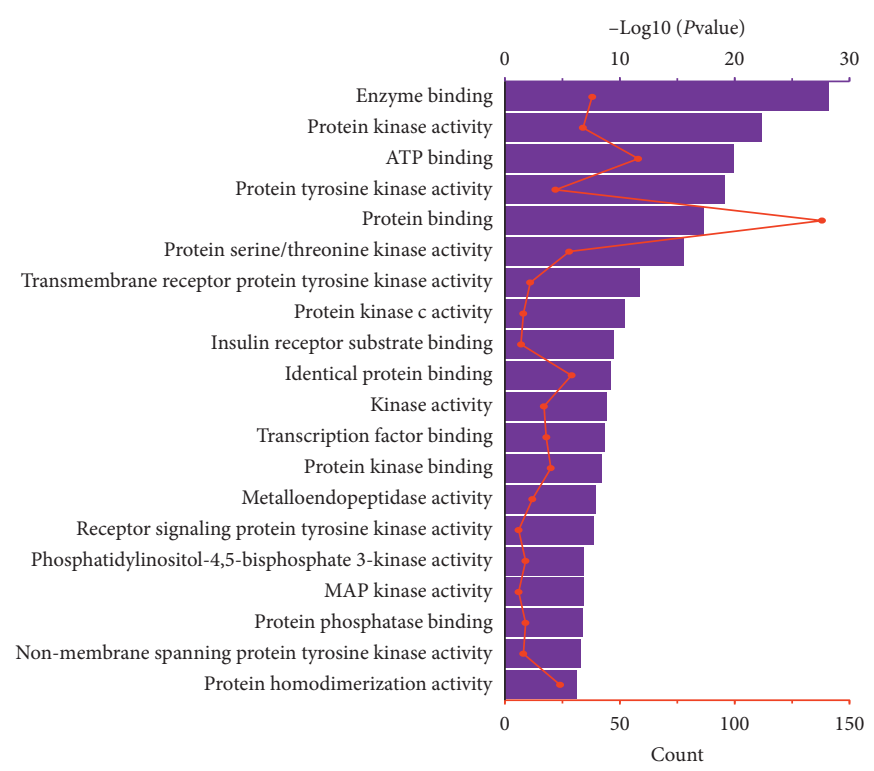

(c)

Figure 5: Results of GO enrichment analysis of the potential targets. (a) Biological processes. (b) Cellular components. (c) Molecular functions. The $\mathrm{y}$-axis shows significantly enriched categories of the targets and the $\mathrm{x}$-axis shows the $-\log 10(P$ value $)$ and enrichment counts of each term $(P<0.05)$.

compound targets with higher docking scores are shown in Figure 8. In the two-dimensional schematic, the dotted line represents the interaction between the compound and the amino acid residues of the protein. The compound and the protein could form different types of interactions such as van der Waals, carbon-hydrogen bonds, conventional hydrogen bonds, and alkyl bonds. For example, when kaempferol bound to VEGFA, kaempferol interacted with TYR104 and PRO59 via carbon-hydrogen bonds, and kaempferol interacted with LEU47 and LYS45 to form a pi-alkyl interaction. Based on these data, we can consider that the interactions are the basis of their biologic activities.

\section{Discussion}

Hypertension is one of the most common cardiovascular diseases, with an increasing prevalence rate worldwide. Vascular remodeling is a significant pathological feature of hypertension, in which the early stage is an adaptive process that eventually becomes maladaptive, damaging target organs such as the heart, brain, and kidneys, and causing the complications of hypertension. TCM has a long history of treating hypertension, with the characteristics of maintaining stable hypotension and protecting target organs with fewer adverse reactions. FT has been used as a TCM for 


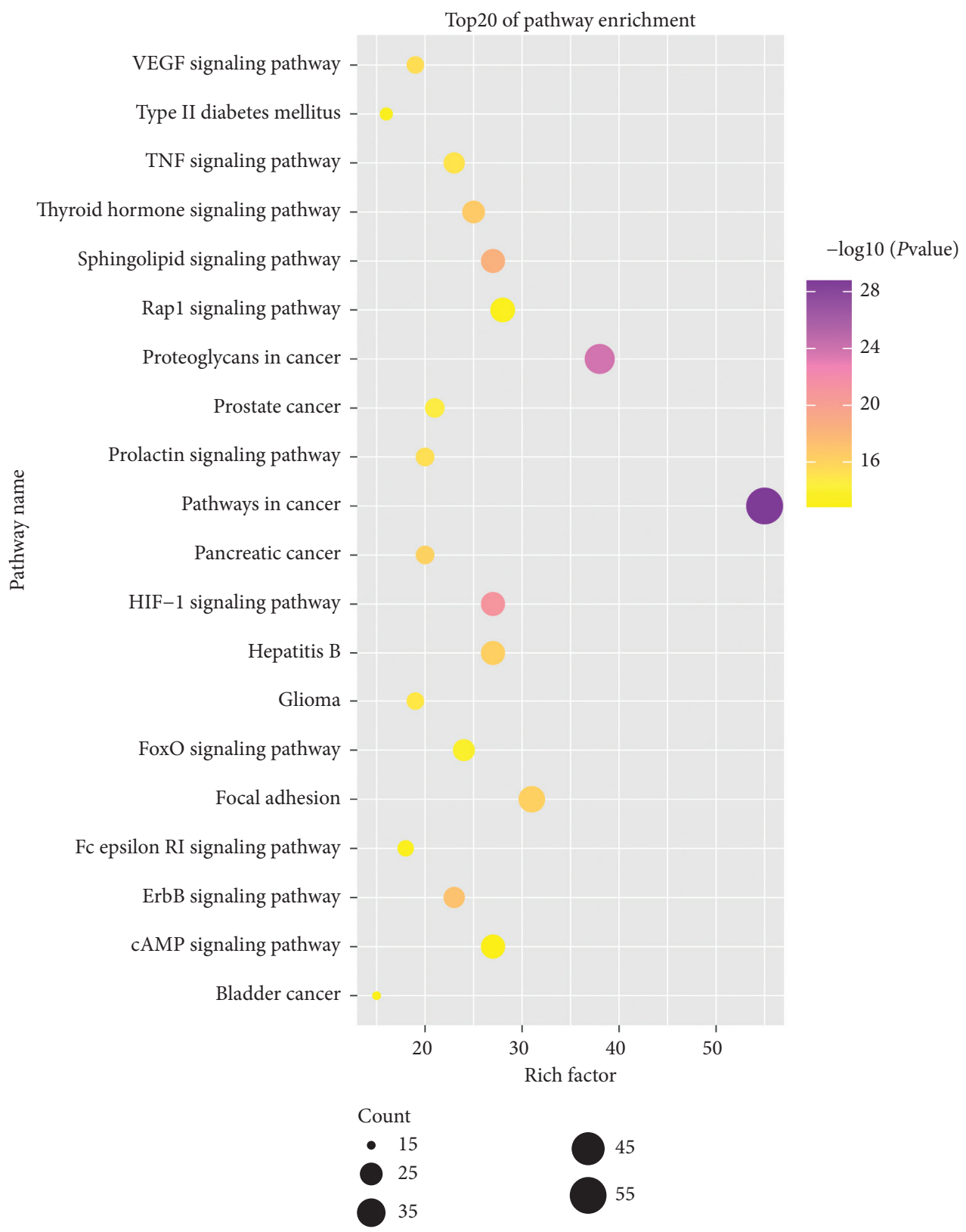

FIGURE 6: Results of KEGG enrichment analysis of the potential targets. The color of the nodes was determined by $-\log 10(P$ value $)$ and the size of the nodes represents the number of counts $(P$ value $<0.05)$.

thousands of years and has a certain effect on the treatment of HVR. Although some preliminary research has been conducted on FT, the molecular targets and mechanisms of FT in treating HVR have not been fully explored. Therefore, elucidating the potential mechanisms is of great significance.

Network pharmacology is a new strategy and method, which helps to investigate the interaction between drugs and diseases and explores the underlying mechanism of a particular therapeutic efficacy. In this study, we constructed a compound-disease target interaction network and found 12 major active compounds. Meanwhile, three topological parameters, degree, betweenness centrality, and closeness certainty were calculated to identify the main targets in the PPI network. Then, GO and KEGG pathway enrichment analyses were carried out to further explore the potential mechanisms of FT in the treatment of HVR. At last, we used molecular docking methods to evaluate the binding activity between the targets and active compounds of FT.

Through the PPI network, we found 33 main target proteins of FT in treating HVR. Among them, the regulation of the MAPK target was of great significance in the treatment of HVR. MAPK mediates a large variety of biological functions including gene expression, cell mitosis, metabolism, motility, survival, apoptosis, and differentiation [35]. One study demonstrated that luteolin could exert effects on HVR by inhibiting angiotensin II-induced proliferation and the migration of vascular smooth muscle cells by regulating the MAPK pathway [36]. VEGFA is a proangiogenic factor 


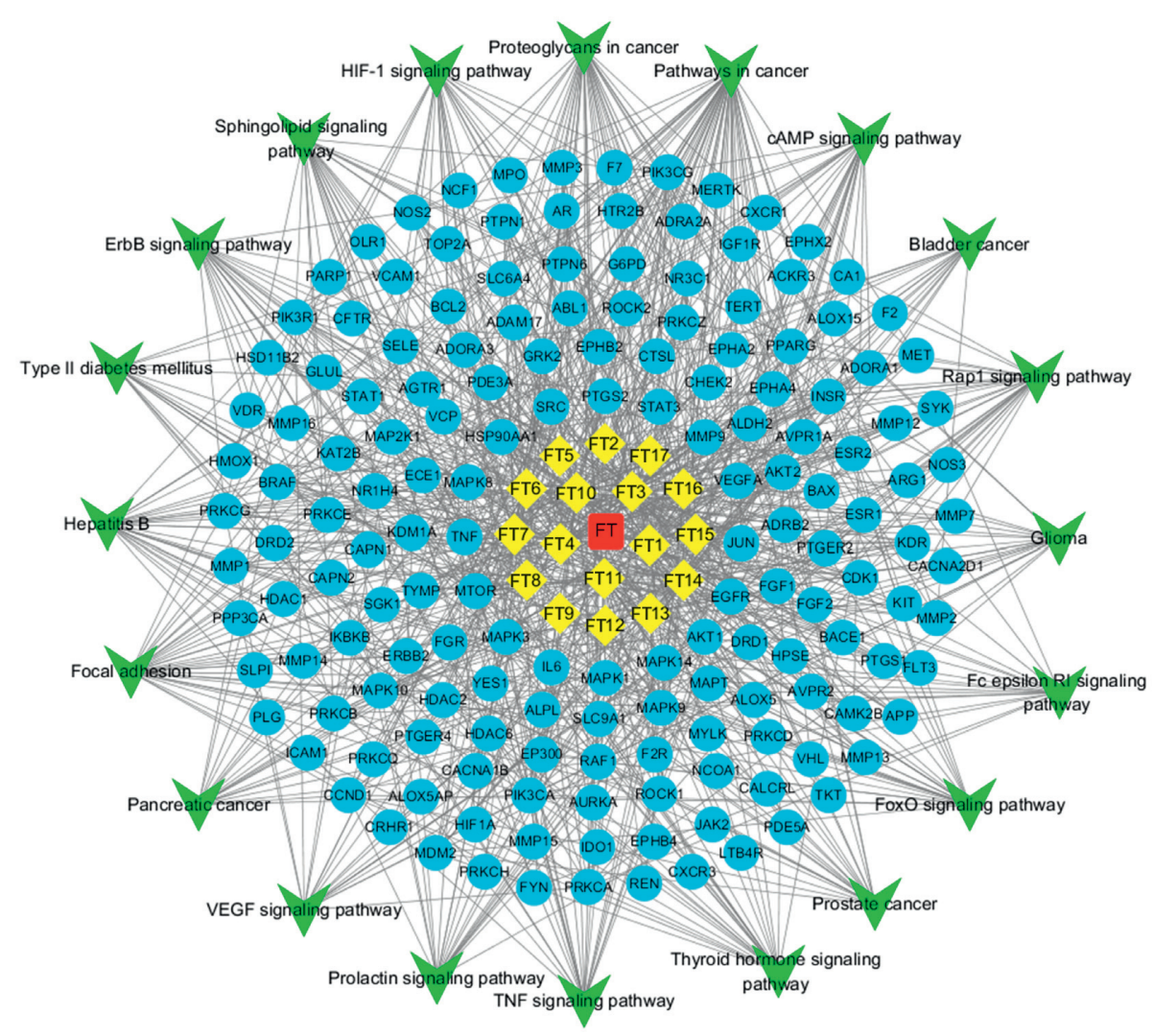

Figure 7: Compound-target-pathway network. The red nodes represent the drug. The yellow nodes represent the compounds. The blue nodes represent the targets. The green nodes represent the pathway of FT in the treatment of HVR. The edges represent the interaction between them.

TABLE 5: LibDock scores of major active compound-main target molecular docking.

\begin{tabular}{|c|c|c|c|c|c|c|}
\hline \multirow{2}{*}{ Number } & \multirow{2}{*}{ Compounds } & \multicolumn{5}{|c|}{ LibDock Score } \\
\hline & & AKT1 & MAPK3 & VEGFA & SRC & IL6 \\
\hline FT3 & Kaempferol & 60.87 & 68.44 & 86.37 & 70.98 & 0.00 \\
\hline FT1 & Isorhamnetin & 60.50 & 112.15 & 123.48 & 92.79 & 67.24 \\
\hline FT11 & $\begin{array}{c}\text { (2aR,2'S,4R,4'R,5'S,6aS,6bS,8aS,8bR,9S, } 11 \mathrm{aR}, 12 \mathrm{aR}, 12 \mathrm{bR})-4,4^{\prime} \text {-dihydroxy-5',6a,8a,9- } \\
\text { tetramethylicosahydro-1H-spiro[pentaleno[2,1-a]phenanthrene-10,2'-pyran]- } \\
8(2 \mathrm{H}) \text {-one }\end{array}$ & 0.00 & 0.00 & 81.20 & 0.00 & 72.51 \\
\hline FT4 & (Z)-3-(4-hydroxy-3-methoxy-phenyl)-N-[2-(4-hydroxyphenyl)ethyl]acrylamide & 94.50 & 110.96 & 94.66 & 99.70 & 85.32 \\
\hline FT10 & Terrestriamide & 0.00 & 0.00 & & 96.77 & 88.63 \\
\hline FT14 & $\begin{array}{c}\text { Tigogenin } \\
(3 \mathrm{R}, 8 \mathrm{~S}, 9 \mathrm{~S}, 10 \mathrm{R}, 13 \mathrm{R}, 14 \mathrm{R}, 17 \mathrm{~S})-17-((2 \mathrm{~S}, 5 \mathrm{R})-5 \text {-ethyl-6-methylheptan-2-yl)- }\end{array}$ & 123.52 & 104.95 & 118.77 & 81.80 & 79.52 \\
\hline FT6 & $\begin{array}{c}\text { 3-hydroxy-10,13-dimethyl-3,4,8,9,10,11,12,13,14,15,16,17-dodecahydro-1H- } \\
\text { cyclopenta } \\
\text { [a]phenanthren-7(2H)-one }\end{array}$ & 127.07 & 62.24 & 116.71 & 110.59 & 83.07 \\
\hline FT8 & (Z)-3-(3,4-dihydroxyphenyl)-N-[2-(4-hydroxyphenyl)ethyl]acrylamide & 90.15 & 107.96 & 93.94 & 89.87 & 88.15 \\
\hline FT13 & Hecogenin & 0.00 & 0.00 & 76.87 & 0.00 & 0.00 \\
\hline FT7 & $\begin{array}{c}\text { (3R,7R,8S,9S,10S,13R,14S,17R)-17-((2R,5S)-5-ethyl-6-methylheptan-2-yl)-3,10- } \\
\text { dimethyl-2,3,4,7,8,9,10,11,12,13,14,15,16,17-tetradecahydro-1H-cyclopenta[a] } \\
\text { phenanthren-7-ol }\end{array}$ & 95.34 & 113.95 & 94.71 & 87.17 & 92.55 \\
\hline FT2 & Sitosterol & 0.00 & 0.00 & 108.59 & 55.55 & 61.00 \\
\hline FT15 & Terrestrosin D & 0.00 & 0.00 & 151.75 & 167.11 & 0.00 \\
\hline- & Ligand & 86.31 & 88.48 & 47.65 & 133.52 & 72.78 \\
\hline
\end{tabular}



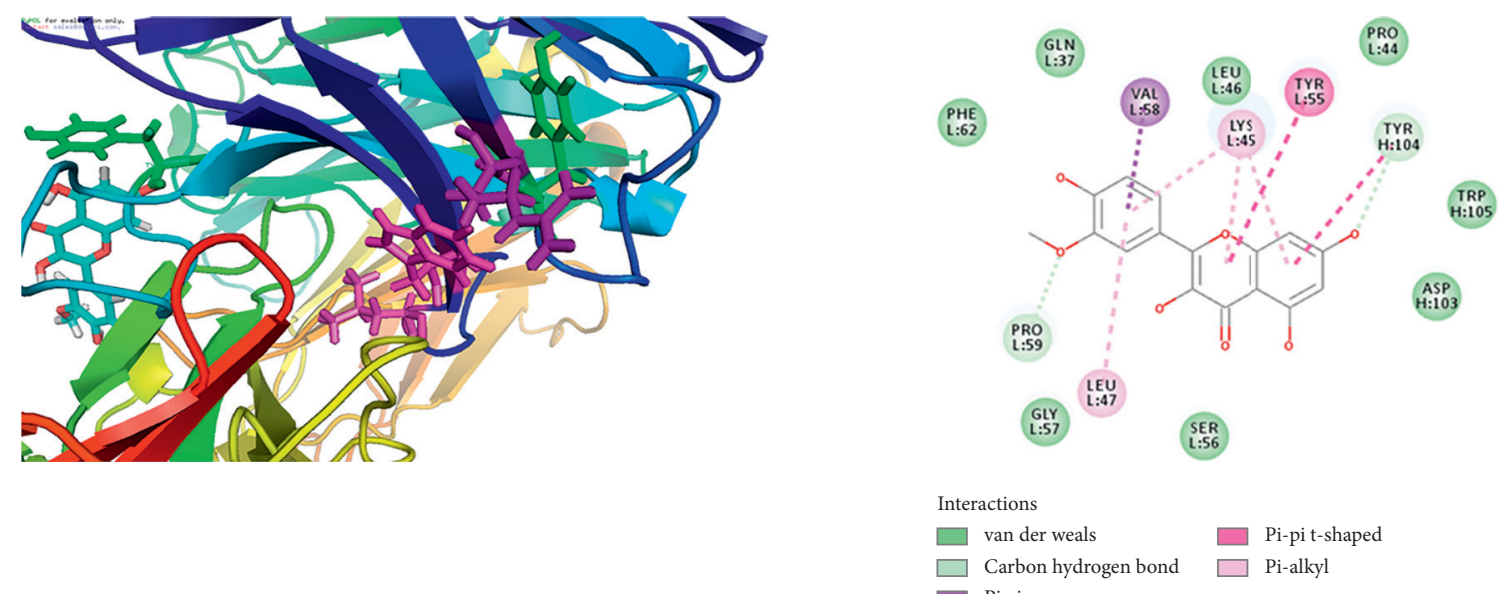

(a)
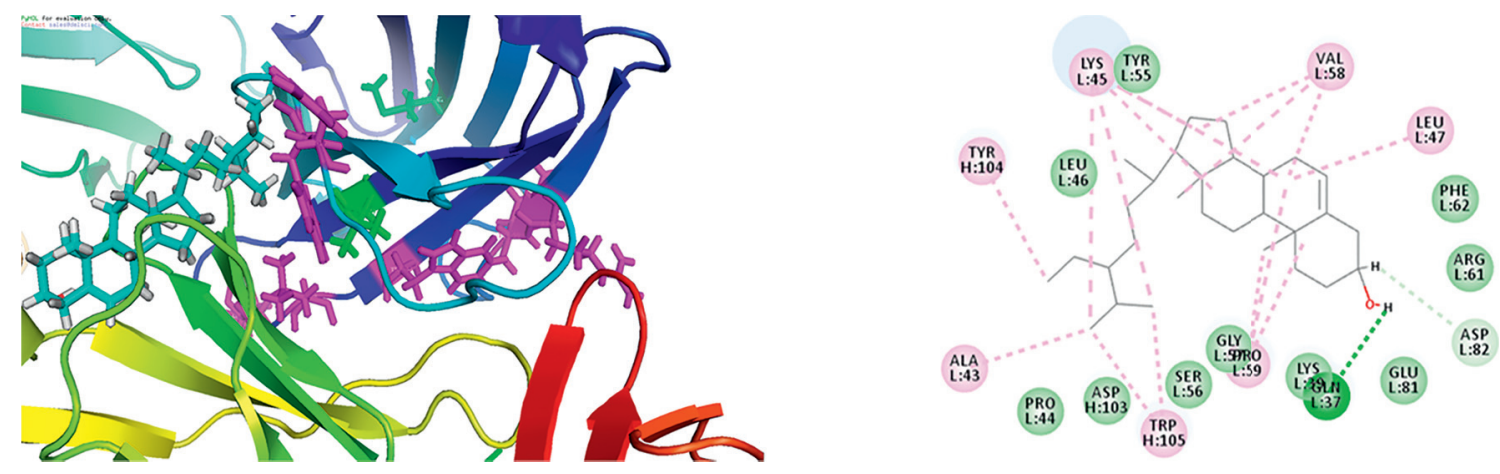

$$
\begin{aligned}
& \text { Interactions } \\
& \square \text { van der weals } \\
& \text { Conventional hydrogen } \quad \square \text { Pi-alkyl } \\
& \text { bond } \\
& \square \text { Carbon hydrogen bond }
\end{aligned}
$$

(b)
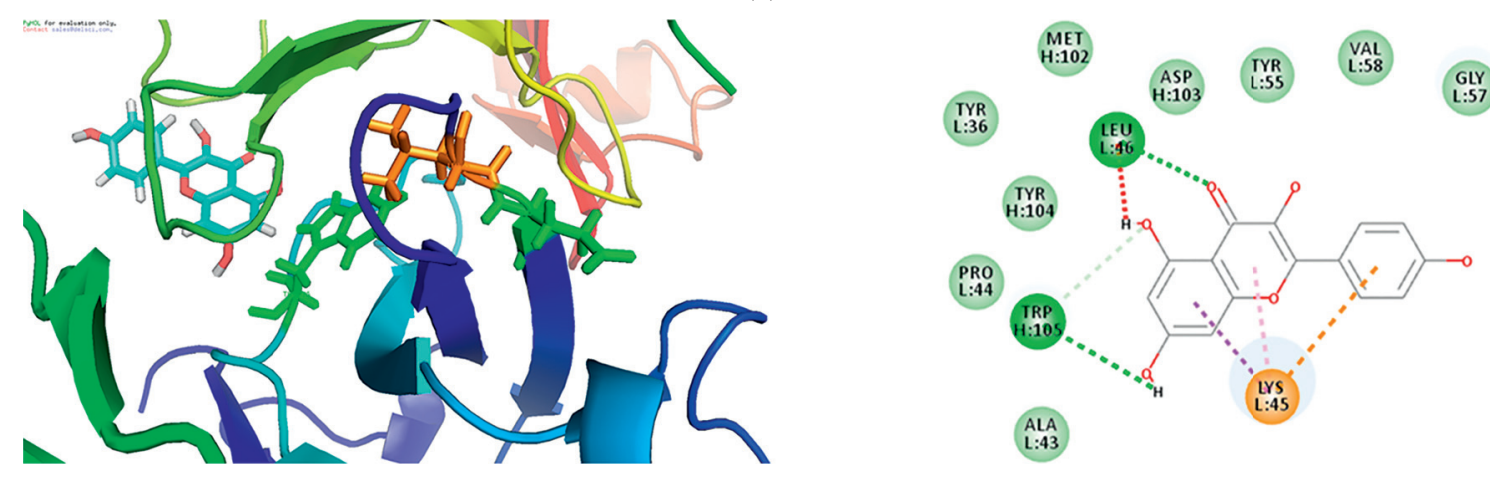

$$
\begin{aligned}
& \square \text { van der weals } \\
& \text { Conventional hydrogen } \\
& \text { bond }
\end{aligned} \begin{aligned}
& \text { Pi-cation } \\
& \text { Pi-signa } \\
& \text { Carbon hydrogen bond }
\end{aligned}
$$

(c)

Figure 8: Continued. 

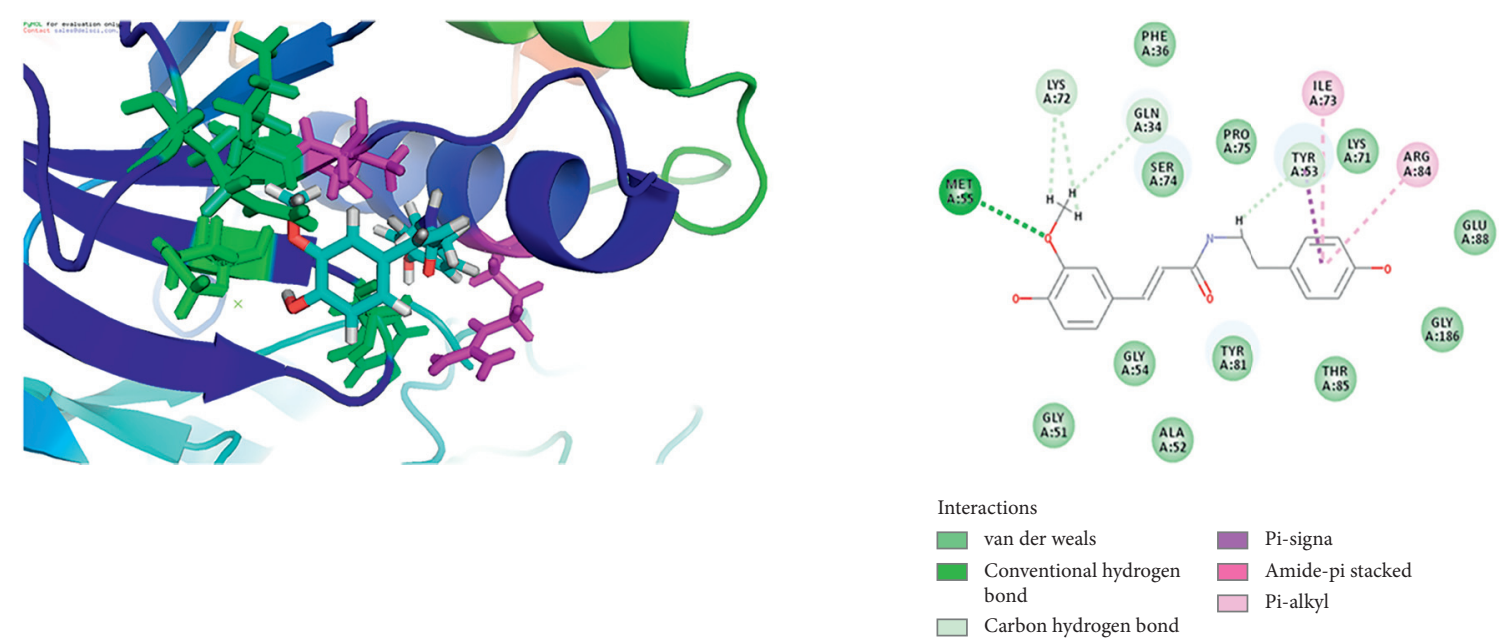

(d)
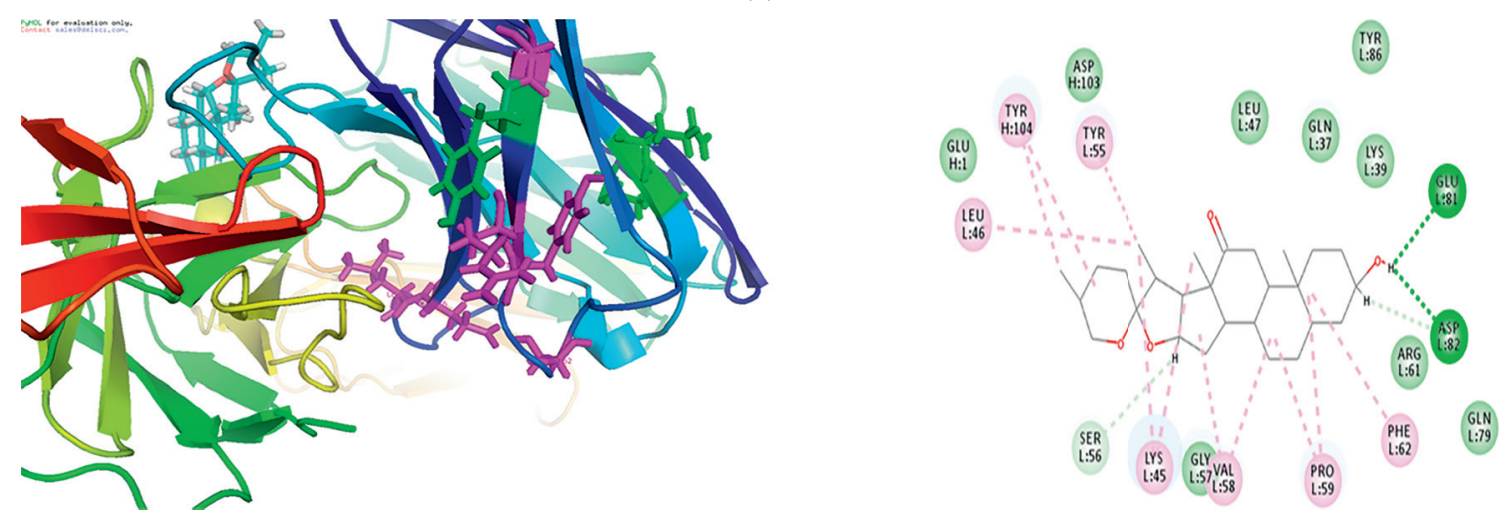

$$
\begin{aligned}
& \text { Interactions } \\
& \square \text { van der weals } \\
& \text { Conventional hydrogen } \quad \square \text { Alkyl } \\
& \text { bond } \\
& \square \text { Carbon hydrogen bond }
\end{aligned}
$$

(e)
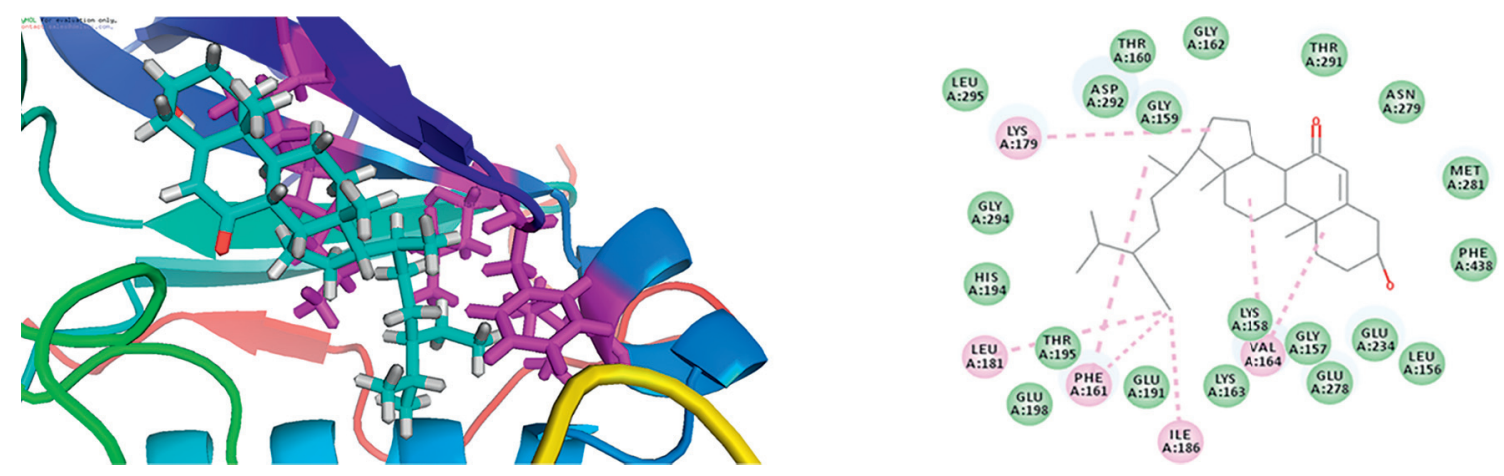

$$
\begin{aligned}
& \text { Interactions } \\
& \square \quad \text { van der weals } \\
& \square \quad \text { Alkyl } \\
& \square \text { Pi-alkyl }
\end{aligned}
$$

(f)

Figure 8: Continued. 

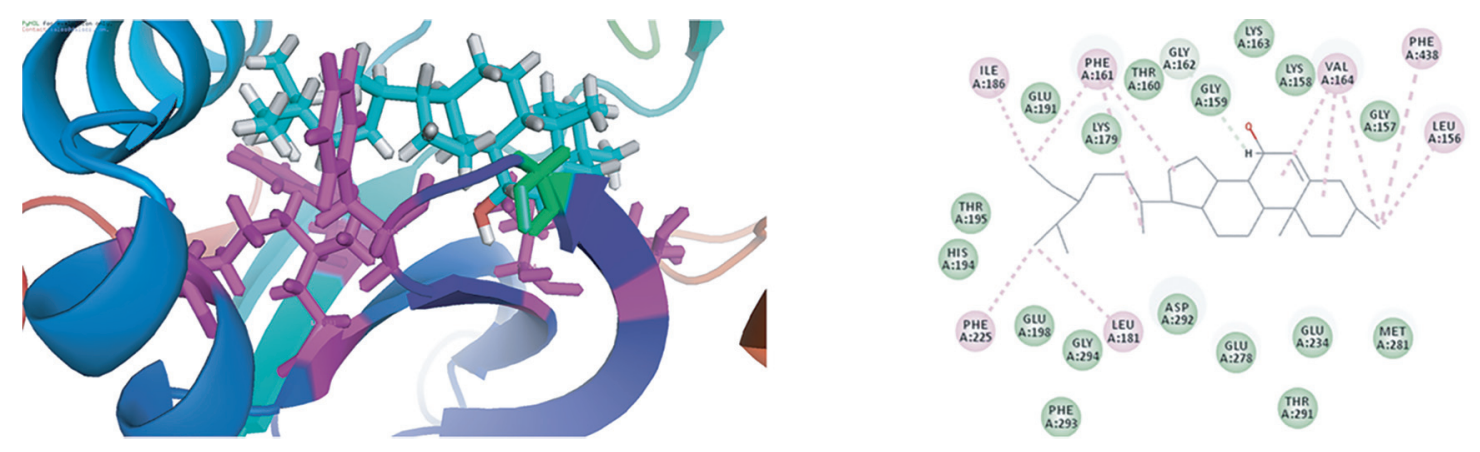

Interactions

$\begin{array}{ll}\square \text { van der weals } & \square \text { Alkyl } \\ \square \text { Carbon hydrogen bond } \quad \square \text { Pi-alkyl }\end{array}$

(g)
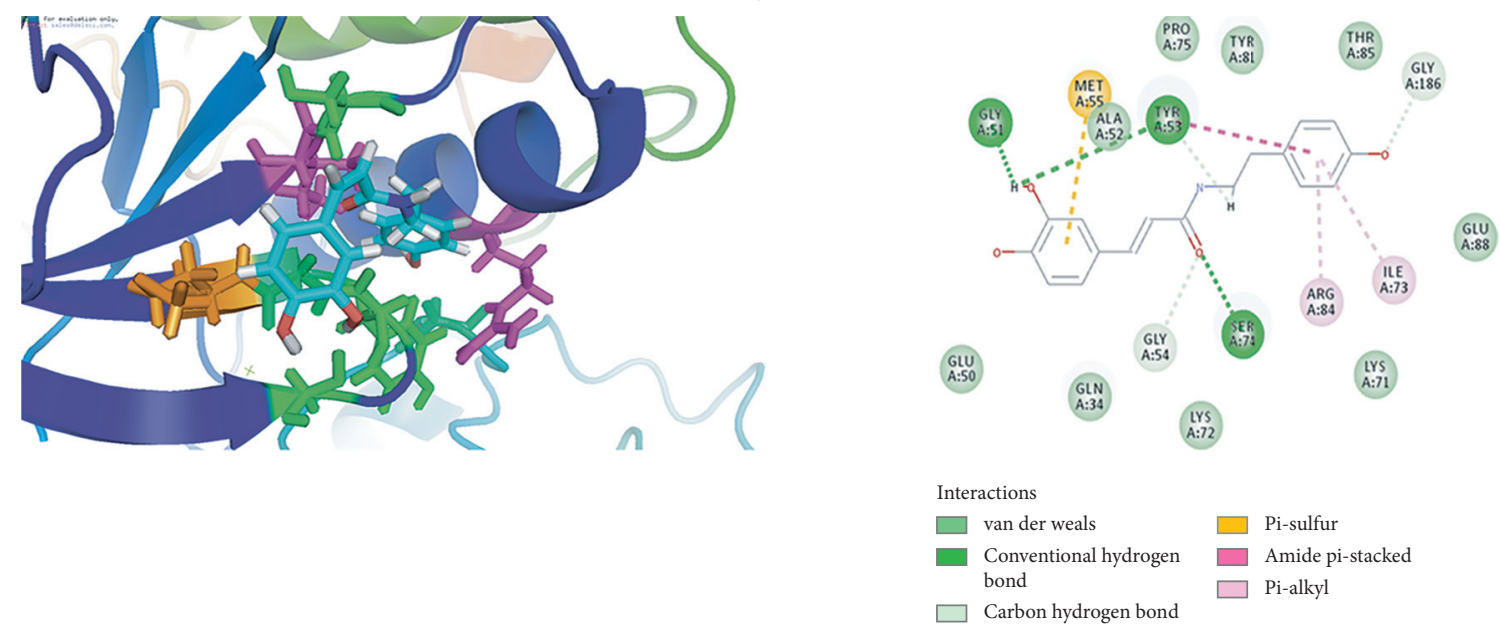

(h)
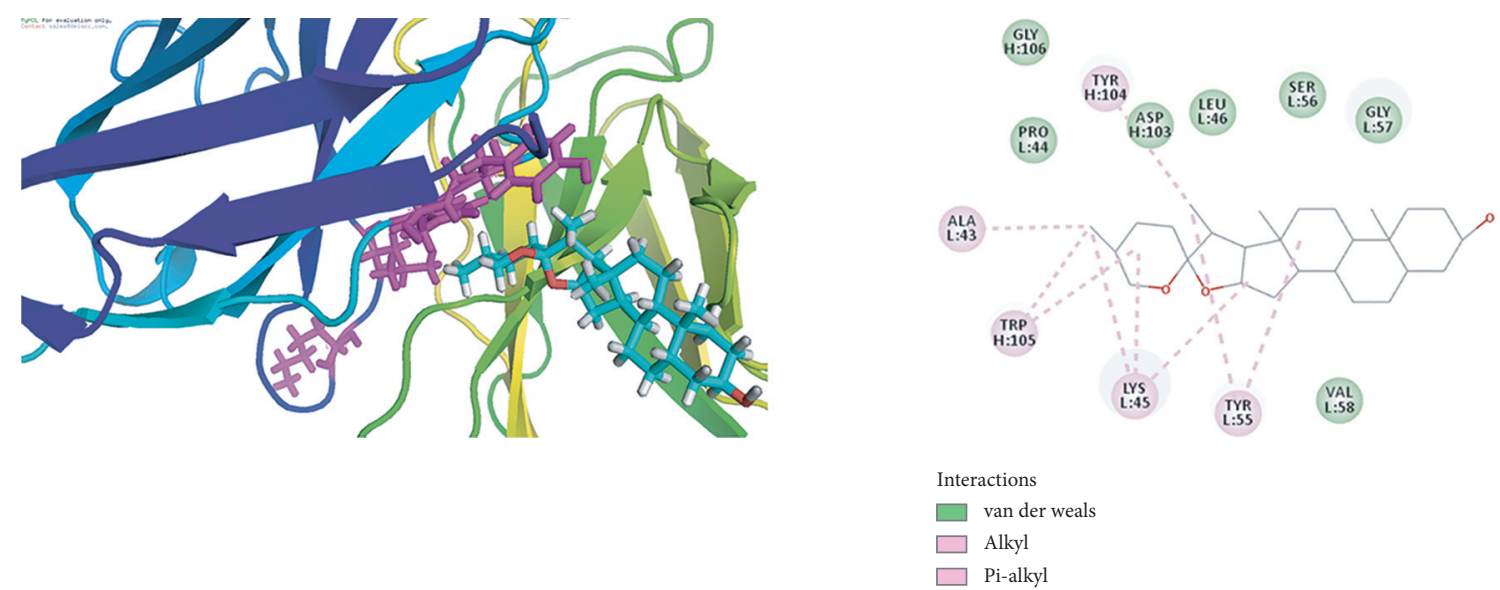

(i)

Figure 8: Continued. 

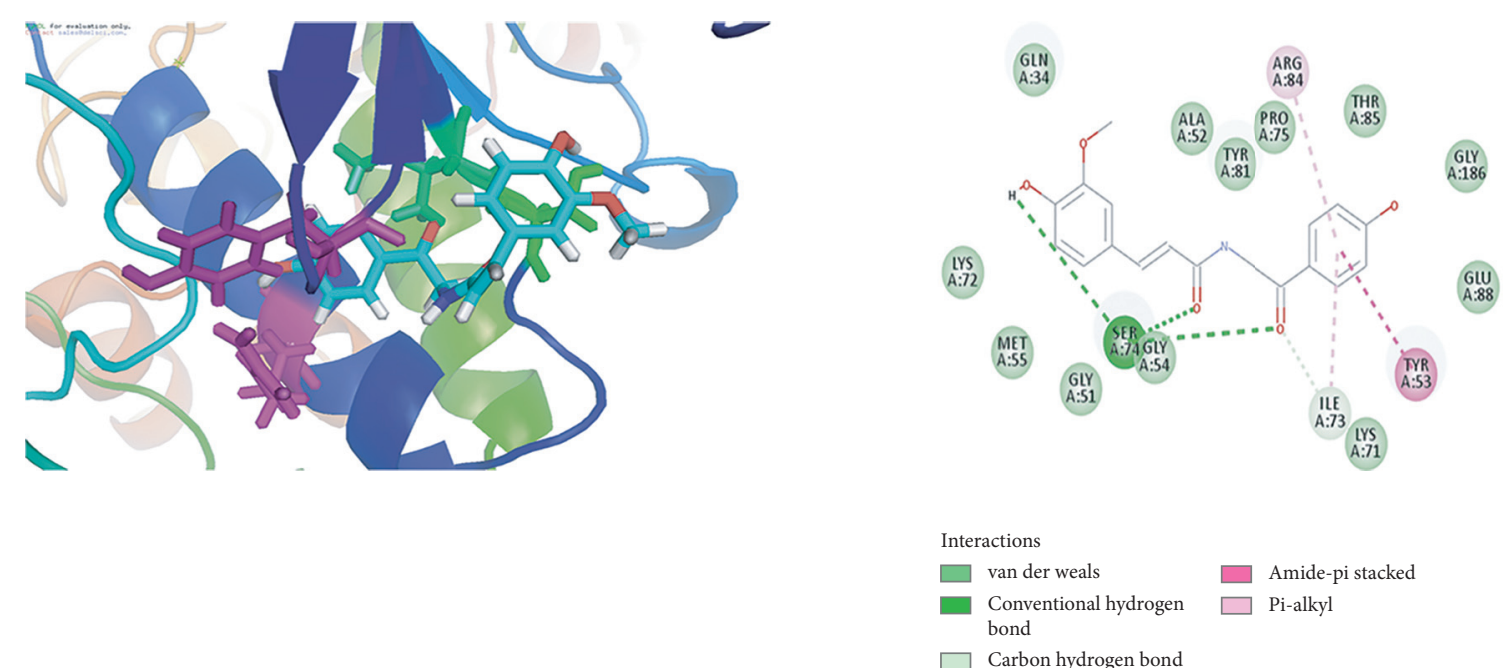

(j)
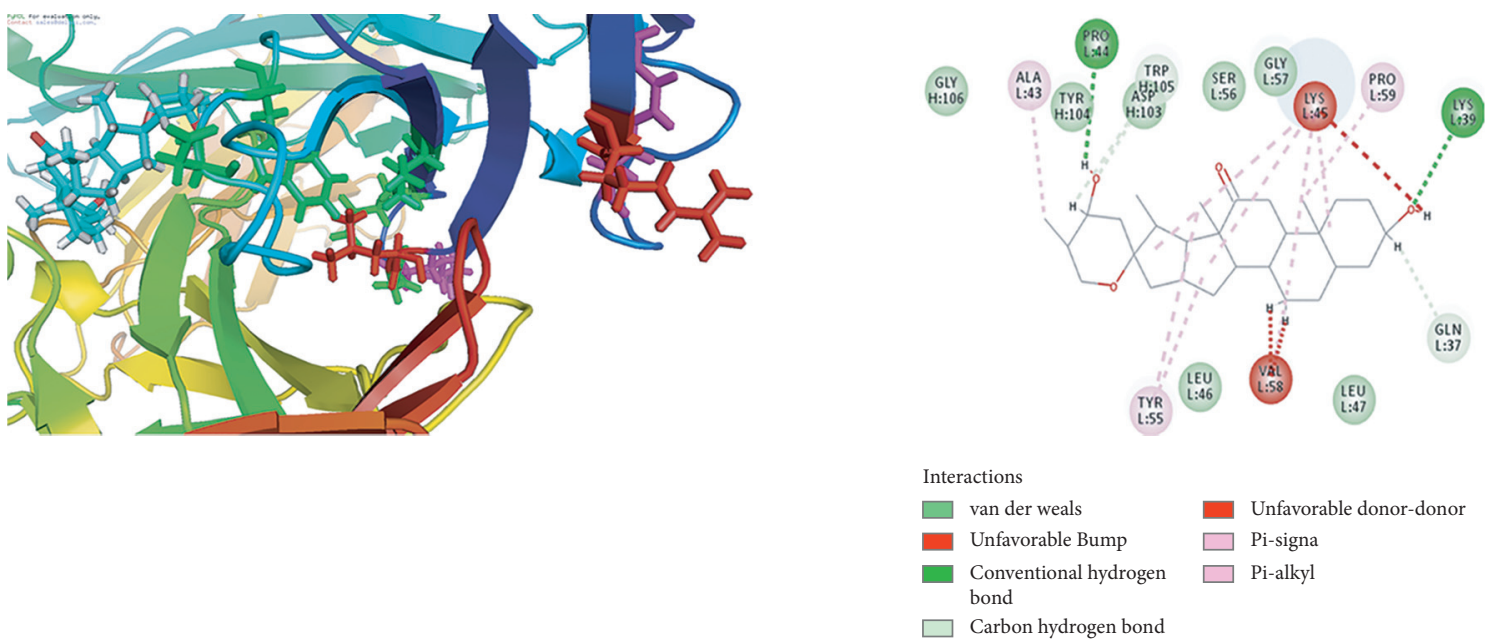

(k)
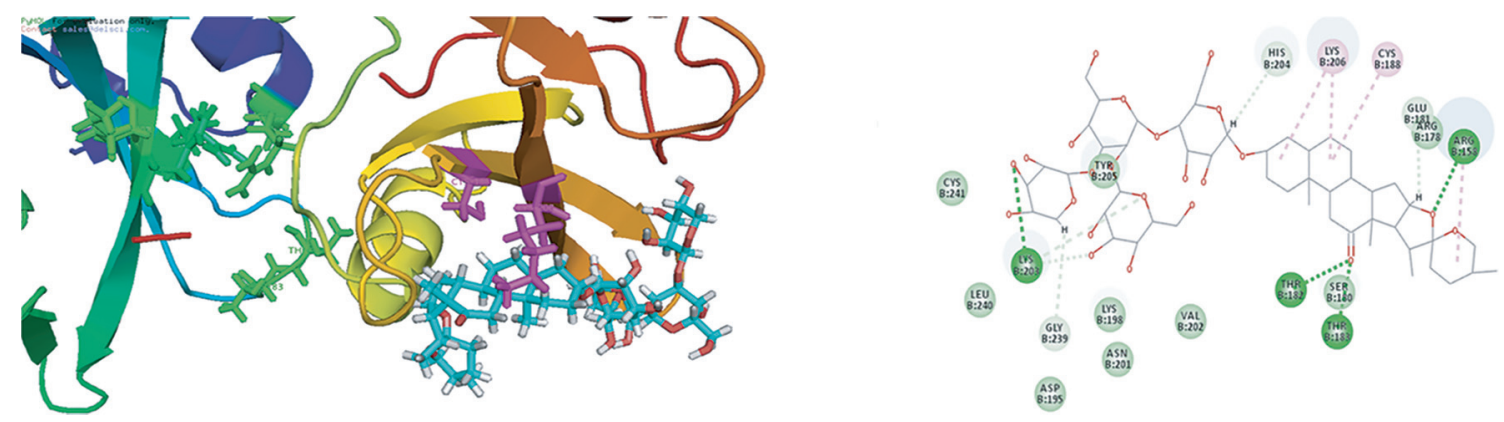

Interactions
$\square$ Van der weals
$\begin{aligned} & \text { Conventional hydrogen } \quad \square \text { Carbon hydrogen bond } \\ & \text { bond }\end{aligned}$

(1)

FIGURE 8: Molecular docking between the major active compounds and main targets. (a) FT3 docking with VEGFA. (b) FT1 docking with VEGFA. (c) FT11 docking with VEGFA. (d) FT4 docking with MAPK3. (e) FT10 docking with VEGFA. (f) FT14 docking with AKT1. (g) FT6 docking with AKT1. (h) FT8 docking with MAPK3. (i) FT13 docking with VEGFA. (j) FT7 docking with MAPK3. (k) FT2 docking with VEGFA. (l) FT15 docking with SRC. 
[37] active in angiogenesis, vasculogenesis, and endothelial cell growth. It was the first member of the VEGF family to be discovered and is the most studied member. Related studies found that the downregulation of VEGFA protein levels could inhibit cell migration and the tube formation of human umbilical vein endothelial cells induced by conditioned medium derived from U251 cell culture [38]. Simultaneously, the VEGF/Flt-1 pathway could enhance the production of inflammatory molecules, chemotactic mediators, and adhesion molecules and induce vascular remodeling in an autocrine or paracrine manner. A previous study demonstrated that reduced VEGF protein expression and its receptor Flt- 1 could decrease neovascularization and improve vascular remodeling [39-41]. IL6 is a pleiotropic cytokine and studies have demonstrated that IL-6 plays a crucial role in the pathophysiology of Takayasu arteritis and giant cell arteritis [42]. Takahiro et al. found that IL-6 blockade by a monoclonal anti-IL-6 receptor antibody could ameliorate hypoxia-induced pulmonary hypertension [43]. According to the literature [44], in supracoronary aortic banding plus metabolic syndrome animals, reducing IL-6, either by anti-IL- 6 antibody or metformin treatment, could reverse pulmonary vascular remodeling.

The KEGG analysis provided a further understanding of the mechanism of HVR. HIF-1 is a transcription factor that regulates the homeostasis of oxygen concentration and is activated under hypoxic conditions, resulting in the upregulation of its target genes, which include VEGF. Many studies have confirmed that the HIF-1 signaling pathway is related to cardiovascular diseases. Mo et al. found that the onset of capillary leakage was associated with an upregulated HIF-1a/VEGFA signaling pathway, causing dysregulation in the immune response and affecting vascular permeability [45]. One cell culture study pointed to endothelial cell HIF signaling as important for connective tissue growth factor expression, vascular permeability, and endothelium-smooth muscle cell interactions that could promote vascular remodeling. In addition, the results demonstrated that endothelial HIF signaling could regulate pulmonary fibrosisassociated pulmonary hypertension [46]. The ErbB signaling pathway was another pathway enriched in our study. The NRG/ErbB system plays a crucial role in the development, maintenance of function, and repair responses to injury in the cardiovascular system. Hedhli et al. summarized that the ErbB signaling pathway is an important mediator of vascular preservation and angiogenic responses in the endothelium [47]. The VEGF signaling pathway fulfills a cardinal role in endothelial cells and its inhibition has profound cardiovascular impacts. TNF is a proinflammatory factor mainly secreted by macrophages. The TNF signaling pathway can participate in the systemic inflammatory response and interact with the NF- $\kappa \mathrm{B}$ and MAPK pathways, leading to the gradual progression of the disease. The FoxO signaling pathway has important roles in cell fate decisions including apoptosis, cell-cycle control, glucose metabolism, oxidative stress resistance, and longevity [48]. These results suggest that targets were involved in many related pathways, demonstrating the multiple compounds, targets, and pathway characteristics of FT in the treatment of HVR and providing a basis for understanding the molecular mechanisms involved.

\section{Conclusion}

Although the efficacy of FT in treating HVR has been confirmed by many studies, the molecular mechanisms of FT remain unclear. In the present study, we screened 12 major active compounds and 33 major targets. Functional enrichment analyses including GO and KEGG pathway analyses were performed, and $645 \mathrm{GO}$ terms and 122 pathways were ascertained. Last, we used molecular docking methods to evaluate the binding activity between the targets and active compounds of FT. This study clarified the multiple compounds, targets, and pathway characteristics of FT in the treatment of HVR and provided a systematic view of the potential mechanisms from the combination of network pharmacology and a literature search.

\section{Abbreviations}

ADME: Absorption, distribution, metabolism, and excretion

BP: $\quad$ Biological processes

CC: $\quad$ Cellular components

DL: Drug-likeness

FT: $\quad$ Fructus Tribuli

GO: $\quad$ Gene ontology

HVR: Hypertensive vascular remodeling

KEGG: Kyoto encyclopedia of genes and genomes

MCODE: Molecular complex detection

MF: $\quad$ Molecular functions

OB: $\quad$ Oral bioavailability

OMIM: Online Mendelian Inheritance in Man

PPI: $\quad$ Protein-protein interaction network

SHRs: Spontaneously hypertensive rats

TCM: Traditional Chinese medicine

TCMSP: Traditional Chinese Medicine Systems Pharmacology Database and Analysis Platform

TSV: Tab-separated value.

\section{Data Availability}

All data generated or analyzed during this study are included within the paper.

\section{Conflicts of Interest}

The authors declare that they have no conflicts of interest.

\section{Authors' Contributions}

Chao Zhang and Huagang Sheng conceived and designed the experiment. Shuyue Wang and Fei Guo analyzed the data and edited the manuscript. Xiaochen Sun, Xiao Song, and Yaohui Yuan were responsible for figure drawing and table design. Guitao Lin revised the manuscript. All authors have read and approved the final manuscript. 


\section{Acknowledgments}

This work was supported by the Key Research and Development Plan of Shandong Province (No. 2019GSF108171), National Natural Science Foundation of China (No. 81603298), and a Project of Shandong Province Traditional Chinese Medicine Science and Technology Development Program (No. 2019-028).

\section{References}

[1] K. T. Mills, J. D. Bundy, T. N. Kelly et al., "Global disparities of hypertension prevalence and control," Circulation, vol. 134, no. 6, pp. 441-450, 2016.

[2] G. Heusch, P. Libby, B. Gersh et al., "Cardiovascular remodelling in coronary artery disease and heart failure," The Lancet, vol. 383, no. 9932, pp. 1933-1943, 2014.

[3] P. Shang, T. Liu, W. Liu et al., "Telmisartan improves vascular remodeling through ameliorating prooxidant and profibrotic mechanisms in hypertension via the involvement of transforming growth factor- $\beta 1$," Molecular Medicine Reports, vol. 16, no. 4, pp. 4537-4544, 2017.

[4] H. Yan and G. Sun, "Research progress on vascular remodeling in hypertension," Chinese Journal of Hypertension, vol. 17, no. 2, pp. 107-109, 2009.

[5] Q. Ma and H. L. Liao, "Research progress of TCM in the treatment of hypertension and vascular remodeling," AsiaPacific Traditional Medicine, vol. 12, no. 2, pp. 58-60, 2016.

[6] Q. Yu, J. C. Zhong, and D. L. Zhu, "RAS system, vascular remodeling and hypertension," Prevention and Treatment of Cardio-Cerebral-Vascular Disease, vol. 10, no. 1, pp. 58-59, 2010.

[7] Chinese Pharmacopoeia Commission, Chinese Pharmacopoeia, China Medical Science Press, Beijing, China, 2020.

[8] S. F. GamalEl Din, M. A. Abdel Salam, M. S. Mohamed et al., "Tribulus terrestris versus placebo in the treatment of erectile dysfunction and lower urinary tract symptoms in patients with late-onset hypogonadism: a placebo-controlled study," Urologia Journal, vol. 86, no. 2, pp. 74-78, 2019.

[9] Y.-H. Jiang, C.-H. Yang, W. Li, S. Wu, X.-Q. Meng, and D.-N. Li, "Aqueous extracts of Tribulus terrestris protects against oxidized low-density lipoprotein-induced endothelial dysfunction," Chinese Journal of Integrative Medicine, vol. 22, no. 3, pp. 193-200, 2016.

[10] Z. Chauhdary, U. Saleem, B. Ahmad, S. Shah, and M. A. Shah, "Neuroprotective evaluation of Tribulus terrestris L. in aluminum chloride induced Alzheimer's disease," Pakistan Journal of Pharmaceutical Sciences, vol. 32, no. 2 (Supplementary), pp. 805-816, 2019.

[11] Z. Wang, D. Zhang, S. Hui, Y. Zhang, and S. Hu, "Effect of Tribulus terrestris saponins on behavior and neuroendocrine in chronic mild stress depression rats," Journal of Traditional Chinese Medicine, vol. 33, no. 2, pp. 228-232, 2013.

[12] N. B. Samani, A. Jokar, M. Soveid, M. Heydari, and S. H. Mosavat, "Efficacy of the hydroalcoholic extract of Tribulus terrestris on the serum glucose and lipid profile of women with diabetes mellitus," Journal of Evidence-Based Complementary \& Alternative Medicine, vol. 21, no. 4, pp. NP91-NP97, 2016.

[13] N. Prabhu, S. Hadigal, U. Sheetal, and A. Shenoy, "Effect of Tribulus terrestris on learning and memory in wistar rats," Pharmacognosy Journal, vol. 6, no. 4, pp. 68-71, 2014.

[14] S. Y. Kang, H. W. Jung, J. H. Nam et al., "Effects of the fruit extract of Tribulus terrestris on skin inflammation in mice with oxazolone-induced atopic dermatitis through regulation of calcium channels, orai-1 and TRPV3, and mast cell activation," Evidence-Based Complementary and Alternative Medicine, vol. 2017, Article ID 8312946, 2017.

[15] H. M. Hammoda, N. M. Ghazy, F. M. Harraz, M. M. Radwan, M. A. ElSohly, and I. I. Abdallah, "Chemical constituents from Tribulus terrestris and screening of their antioxidant activity," Phytochemistry, vol. 92, pp. 153-159, 2013.

[16] Y. Y. Zhang, R. Y. Gu, and G. Y. Wang, "Influences of effective components of Baijili (Fructus Tribuli) on myocardial fibrosis and TGF $\beta 1$ and CTGF expressions in spontaneously hypertension rats," Journal of Beijing University of Traditional Chinese Medicine, vol. 34, no. 2, pp. 111-114, 2011.

[17] J. H. Guo, Y. H. Jiang, C. H. Yang, and Y. C. Liu, "Effect of Tribulus terrestris 1 on thoracic aorta vascular remodeling of spontaneous hypertension rats," Journal of Traditional Chinese Medicine, vol. 57, no. 11, pp. 957-961, 2016.

[18] Y.-H. Jiang, J.-H. Guo, S. Wu, and C.-H. Yang, "Vascular protective effects of aqueous extracts of Tribulus terrestris on hypertensive endothelial injury," Chinese Journal of Natural Medicines, vol. 15, no. 8, pp. 606-614, 2017.

[19] Y. Z. Nie, "Clinical application progress of Xin-Nao-Shu-Tong capsule," Journal of Shandong University of TCM, vol. 33, no. 6, pp. 543-545, 2009.

[20] A. L. Hopkins, "Network pharmacology: the next paradigm in drug discovery," Nature Chemical Biology, vol. 4, no. 11, pp. 682-690, 2008.

[21] H. D. Yuan, Q. Q. Ma, H. Y. Cui et al., "How can synergism of traditional medicines benefit from network pharmacology?" Molecules, vol. 22, no. 7, pp. 1-19, 2017.

[22] J. L. Ru, P. Li, J. N. Wang et al., "TCMSP: a database of systems pharmacology for drug discovery from herbal medicines," Journal of Cheminformatics, vol. 6, no. 13, pp. 1-6, 2014.

[23] M. A. Alam, F. I. Al-Jenoobi, A. M. Al-Mohizea, and R. Ali, "Understanding and managing oral bioavailability: physiological concepts and patents," Recent Patents on Anti-Cancer Drug Discovery, vol. 10, no. 1, pp. 87-96, 2015.

[24] S. Tian, J. M. Wang, Y. Y. Li, D. Li, L. Xu, and T. J. Hou, "The application of in silico drug-likeness predictions in pharmaceutical research," Advanced Drug Delivery Reviews, vol. 86, pp. 1-9, 2015.

[25] J. Lee-Barber, V. Kulo, H. Lehmann, A. Hamosh, and J. Bodurtha, "Bioinformatics for medical students: a 5-year experience using OMIM in medical student education," Genetics in Medicine, vol. 21, no. 2, pp. 493-497, 2019.

[26] M. Safran, V. Chalifa-Caspi, O. Shmueli et al., "Human genecentric databases at the weizmann institute of science: GeneCards, UDB, CroW 21 and HORDE," Nucleic Acids Research, vol. 31, no. 1, pp. 142-146, 2003.

[27] D. S. Wishart and A. Wu, "Using DrugBank for in silico drug exploration and discovery," Current Protocols in Bioinformatics, vol. 54, no. 14, pp. 1-31, 2016.

[28] D. Szklarczyk, A. L. Gable, D. Lyon et al., "STRING v11: protein-protein association networks with increased coverage, supporting functional discovery in genome-wide experimental datasets," Nucleic Acids Research, vol. 47, no. D1, pp. D607-D613, 2019.

[29] M. Franz, C. T. Lopes, G. Huck, Y. Dong, O. Sumer, and G. D. Bader, "Cytoscape.js: a graph theory library for visualisation and analysis," Bioinformatics, vol. 32, no. 2, pp. 309-311, 2016.

[30] C. Piao, Z. Sun, D. Jin et al., "Network pharmacology-based investigation of the underlying mechanism of panax notoginseng treatment of diabetic retinopathy," Combinatorial 
Chemistry \& High Throughput Screening, vol. 23, no. 4, pp. 334-344, 2020.

[31] Y. G. Tao, X. F. Huang, J. Y. Wang, M. R. Kang, L. J. Wang, and S. X. Xian, "Exploring molecular mechanism of huangqi in treating heart failure using network pharmacology," Evidence-Based Complementary and Alternative Medicine, vol. 2020, Article ID 6473745, 2020.

[32] R. Yuan, T. Su, C. Zhang et al., "Study on transformation rules of terrestrosin D in course of Tribuli Fructus stir-frying based on simulated processing technology," China Journal of Chinese Materia Medica, vol. 44, no. 14, pp. 3049-3054, 2019.

[33] R. Yuan, L. L. Wang, L. F. Zhang et al., "Changes and mechanisms of terrestroside B and terrestrosin K in stir-frying Tribuli Fructus," China Journal of Chinese Materia Medica, vol. 44, no. 15, pp. 3297-3304, 2019.

[34] X. Q. Su, C. B. Xia, F. L. Yan et al., "Anti-inflammatory mechanism of epimedii folium in treatment of depression based on network pharmacology and molecular docking," Chinese Journal of Experimental Traditional Medical Formulae, vol. 26, no. 17, pp. 162-169, 2020.

[35] M. Cargnello and P. P. Roux, "Activation and function of the MAPKs and their substrates, the MAPK-activated protein kinases," Microbiology and Molecular Biology Reviews, vol. 75, no. 1, pp. 50-83, 2011.

[36] J. Su, H. T. Xu, J. J. Yu et al., "Luteolin ameliorates hypertensive vascular remodeling through inhibiting the proliferation and migration of vascular smooth muscle cells," Evidence-Based Complementary and Alternative Medicine, vol. 2015, Article ID 364876, 2015.

[37] L. Treps, R. Perret, S. Edmond, D. Ricard, and J. Gavard, "Glioblastoma stem-like cells secrete the pro-angiogenic VEGF-A factor in extracellular vesicles," Journal of Extracellular Vesicles, vol. 6, no. 1, 2017.

[38] Y. Liu, Z.-G. Tang, J.-Q. Yang et al., "Low concentration of quercetin antagonizes the invasion and angiogenesis of human glioblastoma U251 cells," OncoTargets and Therapy, vol. 10, pp. 4023-4028, 2017.

[39] A. K. Stannard, R. Khurana, I. M. Evans, V. Sofra, D. I. R. Holmes, and I. Zachary, "Vascular endothelial growth factor synergistically enhances induction of E-selectin by tumor necrosis factor- $\alpha$," Arteriosclerosis, Thrombosis, and Vascular Biology, vol. 27, no. 3, pp. 494-502, 2007.

[40] X. Q. Zhao, J. Kong, Y. X. Zhao et al., "Gene silencing of TACE enhances plaque stability and improves vascular remodeling in a rabbit model of atherosclerosis," Scientific Reports, vol. 5, 2015.

[41] C. J. Zhang, Y. N. Shi, D. F. Liao, K. Du, and L. Tan, "Molecular mechanism of vascular remodeling in hypertension and Chinese medicine intervention," Acta Physiologica Sinica, vol. 71, no. 2, pp. 235-247, 2019.

[42] H. Yoshifuji, "Pathophysiology of large vessel vasculitis and utility of interleukin-6 inhibition therapy," Modern Rheumatology, vol. 29, no. 2, pp. 287-293, 2019.

[43] T. Hashimoto-Kataoka, N. Hosen, T. Sonobe et al., "Interleukin-6/interleukin-21 signaling axis is critical in the pathogenesis of pulmonary arterial hypertension," Proceedings of the National Academy of Sciences, vol. 112, no. 20, pp. E2677-E2686, 2015.

[44] B. Ranchoux, V. Nadeau, A. Bourgeois et al., "Metabolic syndrome exacerbates pulmonary hypertension due to left heart disease," Circulation Research, vol. 125, no. 4, pp. 449-466, 2019.

[45] L. Mo, G. Xu, C. Wu et al., "Key regulatory effect of activated HIF-1 $\alpha$ /VEGFA signaling pathway in systemic capillary leak syndrome confirmed by bioinformatics analysis," Journal of Computational Biology, vol. 27, no. 6, pp. 914-922, 2020.

[46] A. J. Bryant, R. P. Carrick, M. E. McConaha et al., "Endothelial HIF signaling regulates pulmonary fibrosis-associated pulmonary hypertension," American Journal of Physiology-Lung Cellular and Molecular Physiology, vol. 310, no. 3, pp. L249L262, 2016.

[47] N. Hedhli, A. Kalinowski, and K. S. Russell, "Cardiovascular effects of neuregulin-1/ErbB signaling: role in vascular signaling and angiogenesis," Current Pharmaceutical Design, vol. 20, no. 30, pp. 4899-4905, 2014.

[48] M. Farhan, H. Wang, U. Gaur, P. J. Little, J. Xu, and W. Zheng, "FoxO signaling pathways as therapeutic targets in cancer," International Journal of Biological Sciences, vol. 13, no. 7, pp. 815-827, 2017. 